\title{
LINEAR DECISION TREES, SUBSPACE ARRANGEMENTS, AND MÖBIUS FUNCTIONS
}

\author{
ANDERS BJÖRNER AND LÁSZLÓ LOVÁSZ
}

\section{INTRODUCTION}

Let $P$ be a set in $\mathbb{R}^{n}$. Given a point $x$, we want to test if $x \in P$. Our model of computation is a linear decision tree, a rooted ternary tree $T$ where with each interior node $v$ is associated a linear function $l_{v}(x)=\sum_{i} a_{i} x_{i}+b$, and the three edges connecting $v$ to its descendants are labelled " $<", "="$, and " $>$ ". Starting from the root, we move down the tree; at each internal node $v$, we check whether $l_{v}(x)>=<0$ and follow the appropriately labelled edge. Leaves are labelled YES and NO, and arriving at a leaf we read off the answer to the question "is $x \in P$ ?".

Linear decision trees are sometimes surprisingly powerful devices; we mention here the result that some NP-complete problems like the knapsack problem have polynomial depth linear decision trees [MH]. Lower bound results on the size or depth of linear decision trees usually depend on counting the number of connected components of $P$ or $\mathbb{R}^{n} \backslash P$; this method was introduced by Dobkin and Lipton [DL] and further developed by Steele and Yao [SY], Ben-Or [BO], and others. In this paper we describe topological methods that can be applied to obtain lower bounds also if these sets are connected.

The basic idea of this paper was first explored in [BLY]: the Euler characteristic of $P$ or $\mathbb{R}^{n} \backslash P$ was used to estimate the number of leaves in a linear decision tree for $P$. Here we improve and extend the topological methods of [BLY]. In particular, we show that the Euler characteristic can be replaced by the sum of the Betti numbers of $\mathbb{R}^{n} \backslash P$.

In [BLY] the method was applied to the case when $P$ is a subspace arrangement, i.e., a union of a finite number of affine subspaces. The Euler characteristic of the union is (essentially) the Möbius function of the intersection lattice of these subspaces. In this paper, using a formula of Goresky and MacPherson [GM], we work with the Betti numbers of the complement of this union and thereby obtain better bounds.

The motivating problem in [BLY] was the $k$-equal-problem: given $n$ real numbers $x_{1}, \ldots, x_{n}$, decide if some $k$ of them are equal. It was shown that every linear decision tree for the $k$-equal-problem has size at least $(2 n / k)^{\Omega(n)}$ and (consequently) depth at least $\Omega(n \log (2 n / k))$ (which is best possible). We

Received by the editors March 16, 1993 and, in revised form, May 12, 1993.

1991 Mathematics Subject Classification. Primary 05E99, 06A07, 57N99, 68Q25, 68R05. 
improve the constant in this asymptotic bound and give here other applications; in particular, we determine the linear decision tree complexity of the $k$-unequal-problem (given $n$ real numbers $x_{1}, \ldots, x_{n}$, decide if some $k$ of them are pairwise different), and the $k$-divisibility problem (given $n$ real numbers $x_{1}, \ldots, x_{n}$, decide if the number of times any number occurs in this list is divisible by $k$ ).

These applications involve the exact computation of the Möbius function for various subposets of the partition lattice of a finite set. The Möbius function computations are done in a more general setting than what is necessary for the computer science applications and may have some interest in their own right. From these computations is deduced a formula for the Euler characteristic of the subset of $\mathbb{R}^{n}$ of points with no $k$ coordinates equal in terms of the roots of truncated exponential series (see Theorem 6.3).

We remark that Yao [Y] has extended the Euler characteristic bounds given in [BLY] to algebraic decision trees. It is possible that the Betti number bounds can also be extended this way, but the technical difficulties seem to be substantial.

\section{LINEAR DECISION TREES FOR POLYHEDRA}

A convex polyhedron is the solution set of a finite system of (strict and nonstrict) linear inequalities (equivalently, the intersection of a finite number of (open or closed) half-spaces). A polyhedron is the union of a finite number of convex polyhedra. It is clear that a set $P \subseteq \mathbb{R}^{n}$ can be recognized by a linear decision tree if and only if it is a polyhedron.

Suppose that $T$ is a linear decision tree deciding membership in $P$. We denote the number of YES-leaves and NO-leaves by $\ell^{+}(T)$ and $\ell^{-}(T)$, respectively, and we denote by $\ell^{+}(P)$ and $\ell^{-}(P)$ the minimum of $\ell^{+}(T)$ and $\ell^{-}(T)$ over all linear decision trees for $P$.

We will assume some familiarity with cell complexes and elementary homology theory; all needed facts can be found in, e.g., Munkres [Mu]. Let $\chi(X)$ denote the Euler characteristic of a space $X$. We shall use this notion only for spaces $X$ with finite Betti numbers (in fact, having the homotopy type of a finite simplicial complex), so no problem will arise whether $\chi(X)$ is well defined.

In [BLY] the following lower bounds on the numbers of YES- and NO-leaves of any linear decision tree for a closed polyhedron $P$ were proved. Here and in the following we compactify the space $\mathbb{R}^{n}$ by adding a single point $\omega$ "at infinity", and let $\hat{P}=P \cup\{\omega\}$ for any polyhedron $P$.

Theorem 2.1. Let $P$ be a closed polyhedron in $\mathbb{R}^{n}$. Then

$$
\ell^{+}(P) \geq|\chi(\hat{P})-1|, \quad \ell^{-}(P) \geq\left|\chi(\hat{P})-1+(-1)^{n-1}\right| .
$$

We are going to prove much stronger estimates below; however, it seems worthwhile to include the simple direct proof of these Euler characteristic bounds. Note that if $P$ is bounded then $\chi(P)=\chi(\hat{P})-1$, so that for a compact polyhedron we get the inequalities

$$
\ell^{+}(P) \geq|\chi(P)|, \quad \ell^{-}(P) \geq\left|\chi(P)+(-1)^{n-1}\right| .
$$

Proof. Let $T$ be a linear decision tree for $P$, and let $L^{-}$and $L^{+}$be the sets of NO-leaves and YES-leaves, respectively. For each $w \in L=L^{-} \cup L^{+}$, let $P_{w}$ 
denote the set of inputs leading to leaf $w$. Each set $P_{w}$ is a convex subset of $\mathbb{R}^{n}$. Clearly $P$ is the union of all cells $P_{w}\left(w \in L^{+}\right)$.

Each set $P_{w}(w \in L)$ is a convex polyhedron, but not necessarily closed. In fact, $P_{w}$ is open in its affine hull; the affine hull $A_{w}$ of $P_{w}$ is obtained as the intersection of those hyperplanes $l_{u}(x)=0$ which tested with equality along the path from the root to $w$, and the remaining strict inequalities along this path define $P_{w}$. We denote by $\bar{P}_{w}$ the closure of $P_{w}$ in $A_{w}$, and by $\partial P_{w}$ the boundary of $P_{w}$ in $A_{w}$. If $P_{w}$ is bounded, then $\bar{P}_{w}$ is a ball (in fact a convex polytope) and $\partial P_{w}$ is its bounding sphere. If $P_{w}$ is unbounded then there are two cases:

(i) $\hat{\bar{P}}_{w}$ is a ball and $\widehat{\partial P}_{w}$ is its bounding sphere, if $P_{w} \neq A_{w}$,

(ii) $\hat{\bar{P}}_{w}$ is a sphere and $\widehat{\partial P}_{w}=\{\omega\}$, if $P_{w}=A_{w}$.

We have $P=\bigcup\left\{P_{w}: w \in L^{+}\right\}$; but the polyhedra $\bar{P}_{w}$ (even together with their faces) do not form a $\mathrm{CW}$ cell decomposition in general. So to relate to the Euler characteristic, we consider the following finer decomposition. Our linear decision tree $T$ determines an arrangement of affine hyperplanes $\mathscr{A}_{T}=\left\{H_{u}\right\}$, where $H_{u}=\left\{x \in \mathbb{R}^{n}: l_{u}(x)=0\right\}$ for each inner node $u$. These hyperplanes subdivide $\mathbb{R}^{n}$ into a number of relatively open convex (polyhedral) regions. These regions, together with their faces, partition $\mathbb{R}^{n}$ (points in the same class behave the same way in all tests on the tree). Let $\Delta$ denote the set of these classes. Their closures in $\widehat{\mathbb{R}^{n}}$ together with the point $\omega$ give a regular $\mathrm{CW}$ decomposition of $\widehat{\mathbb{R}^{n}}=S^{n}$.

For each leaf $w$, let $\Delta_{w}, \bar{\Delta}_{w}$, and $\partial \Delta_{w}$ be the collections of cells in $\Delta$ contained in $P_{w}, \bar{P}_{w}$, and $\partial P_{w}$, respectively. Let $\Delta_{P}$ denote the set of cells in $\Delta$ contained in $P$ (note that $\sigma \cap P \neq \varnothing$ implies $\sigma \subseteq P$ for all cells $\sigma \in$ $\Delta)$. Then the closures of cells in $\Delta_{P}$ together with $\omega$ form a regular CW decomposition of $\hat{P}$, and hence

$$
\sum_{C \in \Delta_{P}}(-1)^{\operatorname{dim}(C)}=\chi(\hat{P})-1 .
$$

(The "minus 1 " comes from counting $\omega$. .)

Now we can partition this sum according to the YES-leaves:

$$
\begin{aligned}
\chi(\hat{P})-1 & =\sum_{w \in L^{+}} \sum_{C \in \Delta_{w}}(-1)^{\operatorname{dim}(C)}=\sum_{w \in L^{+}}\left(\sum_{C \in \bar{\Delta}_{w}}(-1)^{\operatorname{dim}(C)}-\sum_{C \in \partial \Delta_{w}}(-1)^{\operatorname{dim}(C)}\right) \\
& =\sum_{w \in L^{+}}\left(\left(\chi\left(\hat{\bar{P}}_{w}\right)-1\right)-\left(\chi\left(\widehat{\partial P}_{w}\right)-1\right)\right)=\sum_{w \in L^{+}}(-1)^{\operatorname{dim}\left(P_{w}\right)} .
\end{aligned}
$$

In fact, $\bar{\Delta}_{w} \cup\{\omega\}$ forms a regular CW decomposition of $\hat{\bar{P}}_{w}, \partial \Delta_{w} \cup\{\omega\}$ forms a regular $\mathrm{CW}$ decomposition of $\widehat{\partial P_{w}}$, and if $P_{w}$ is unbounded then $\hat{\bar{P}}_{w}$ and $\widehat{\partial P}_{w}$ are a ball and a sphere (or conversely) as stated in (i) or (ii) above. In the bounded case we get a ball or a sphere plus the isolated point $\omega$. Therefore, $\chi\left(\hat{\bar{P}}_{w}\right)-\chi\left(\widehat{\partial P}_{w}\right)=(-1)^{\operatorname{dim}\left(P_{w}\right)}$ follows in all cases from $\chi($ ball $)=1$ and $\chi(n$-sphere $)=1+(-1)^{n}$. 
The bound on $\ell^{+}$follows immediately from this equation, since the righthand side is at most $\left|L^{+}\right|=\ell^{+}(T)$.

For $\ell^{-}$, observe that the fact that $\Delta \cup\{\omega\}$ forms a $\mathrm{CW}$ decomposition of the $n$-sphere gives $\sum_{C \in \Delta}(-1)^{\operatorname{dim}(C)}=\chi\left(S^{n}\right)-1$. Hence

$$
\sum_{C \in \Delta \backslash \Delta_{P}}(-1)^{\operatorname{dim}(C)}=\chi\left(S^{n}\right)-1-(\chi(\hat{P})-1)=-\chi(\hat{P})+1+(-1)^{n} .
$$

From here the argument is just like that for $\ell^{+}$, by partitioning the left-hand sum according to the NO-leaves.

To be able to state our more involved results linking the size of linear decision trees to Betti numbers, let us assume that an open set $\Omega \subseteq \mathbb{R}^{n}$ is also given. We want to test points in $\Omega$ for membership in a set $P \subseteq \mathbb{R}^{n}$. Let $T$ be a linear decision tree for this problem, and let $P_{w}$ denote the set of inputs $x \in \Omega$ leading to leaf $w$. The dimension of $P_{w}$ is well defined, since it is open in its affine span. Let $L^{-}$be the set of NO-leaves and $L^{+}$the set of YES-leaves of $T$, and define:

$$
\begin{aligned}
& \ell_{i}^{-}(T)=\left|\left\{w \in L^{-}: \operatorname{dim}\left(P_{w}\right)=i\right\}\right| ; \\
& \ell^{-}(T)=\left|L^{-}(T)\right|=\sum_{i} \ell_{i}^{-}(T) ; \\
& \ell_{i}^{+}(T)=\left|\left\{w \in L^{+}: \operatorname{dim}\left(P_{w}\right)=i\right\}\right| ; \\
& \ell^{+}(T)=\left|L^{+}(T)\right|=\sum_{i} \ell_{i}^{+}(T) .
\end{aligned}
$$

Furthermore, for given subsets $P, \Omega \subseteq \mathbb{R}^{n}$, define

$$
\ell_{i}^{-}(P \mid \Omega)=\min _{T} \ell_{i}^{-}(T), \quad \ell_{i}^{+}(P \mid \Omega)=\min _{T} \ell_{i}^{+}(T),
$$

where the minimum is taken over all linear decision trees $T$ that decide for points in $\Omega$ whether they belong to $P$. Define $\ell^{-}(P \mid \Omega)$ and $\ell^{+}(P \mid \Omega)$ analogously. Note that $\ell^{-}(P \mid \Omega) \geq \sum_{i} \ell_{i}^{-}(P \mid \Omega)$. When $\Omega=\mathbb{R}^{n}$, we will suppress $\Omega$ from the notation, and write $\ell^{-}(P)=\ell^{-}(P \mid \Omega)$, etc.

We denote by $\beta_{i}(X)$ the $i$ th Betti number of the subset $X \subseteq \mathbb{R}^{n}$, i.e., the rank of the $i$ th singular homology group $H_{i}(X, \mathbb{Z})$, and by $\tilde{\beta}_{i}(X)$ the $i$ th Betti number in reduced singular homology. We also set $\beta(X)=\sum_{i \geq 0} \beta_{i}(X)$, and $\tilde{\beta}(X)=\sum_{i \geq-1} \tilde{\beta}_{i}(X)$. Then $\beta(X)=1+\tilde{\beta}(X)$ if $X \neq \varnothing$. Our main result in this section is the following topological lower bound on the number of $i$-dimensional leaves.

Theorem 2.2. Let $P$ be a closed polyhedron and $\Omega$ a convex open set in $\mathbb{R}^{n}$. Then

and

$$
\ell_{n-i}^{-}(P \mid \Omega) \geq \beta_{i}(\Omega \backslash P),
$$

$$
\ell_{n-i}^{+}(P \mid \Omega) \geq \tilde{\beta}_{i-1}(\Omega \backslash P) \text {. }
$$

Proof. Let $T$ be a linear decision tree testing points in $\Omega$ for membership in $P$. We will show that

$$
\ell_{n-i}^{-}(T) \geq \beta_{i}(\Omega \backslash P)
$$


by induction on the number of nodes of $T$. It is straightforward to check the case when $T$ has a single node; the case of a single NO-leaf uses that $\Omega$ is both convex (for $\beta_{0}=1$ ) and open (for $\ell_{n}^{-}=1$ ).

Assume that $T$ has more than one node, and let $q(x)$ be the linear form used at the root node. This divides $\Omega$ into three parts:

$$
\begin{aligned}
\Omega^{+} & =\{x \in \Omega \mid q(x)>0\}, \\
\Omega^{-} & =\{x \in \Omega \mid q(x)<0\}, \\
\Omega^{0} & =\{x \in \Omega \mid q(x)=0\} .
\end{aligned}
$$

All three sets are nonempty (unless the tree is degenerate), and points from these subsets are sent to the three subtrees $T^{+}, T^{-}$, and $T^{0}$ rooted at the sons of the root. Note that $T^{i}$ is a linear decision tree for $P$ and $\Omega^{i}$ in $\mathbb{R}^{n}$, for $i=+,-$, and $T^{0}$ is a linear decision tree for $P \cap H$ and $\Omega^{0}$ in $H=\left\{x \in \mathbb{R}^{n} \mid q(x)=0\right\} \cong \mathbb{R}^{n-1}$. The induction assumption gives that

$$
\begin{aligned}
\ell_{n-i}^{-}(T) & =\ell_{n-i}^{-}\left(T^{+}\right)+\ell_{n-i}^{-}\left(T^{-}\right)+\ell_{n-i}^{-}\left(T^{0}\right) \\
& \geq \ell_{n-i}^{-}\left(P \mid \Omega^{+}\right)+\ell_{n-i}^{-}\left(P \mid \Omega^{-}\right)+\ell_{(n-1)-(i-1)}^{-}\left(P \cap H \mid \Omega^{0}\right) \\
& \geq \beta_{i}\left(\Omega^{+} \backslash P\right)+\beta_{i}\left(\Omega^{-} \backslash P\right)+\beta_{i-1}\left(\Omega^{0} \backslash P\right) .
\end{aligned}
$$

The proof will be concluded by showing that

$$
\beta_{i}(\Omega \backslash P) \leq \beta_{i}\left(\Omega^{+} \backslash P\right)+\beta_{i}\left(\Omega^{-} \backslash P\right)+\beta_{i-1}\left(\Omega^{0} \backslash P\right) .
$$

For each point $x \in \Omega^{0} \backslash P$, choose an open ball $O_{x}$ centered at $x$ such that $O_{x} \subseteq \Omega \backslash P$ (which is an open set in $\mathbb{R}^{n}$ ). Let $C=\bigcup_{x \in \Omega^{0} \backslash P} O_{x}$. Orthogonal projection onto $H$ gives rise to the homotopy equivalence

$$
C \simeq \Omega^{0} \backslash P
$$

(in fact, $\Omega^{0} \backslash P$ is a strong deformation retract of $C$ ). Let $A^{+}=\left(\Omega^{+} \backslash P\right) \cup C$ and $A^{-}=\left(\Omega^{-} \backslash P\right) \cup C$. Then we have homotopy equivalences

$$
A^{+} \simeq \Omega^{+} \backslash P, \quad A^{-} \simeq \Omega^{-} \backslash P,
$$

since $\left(\Omega^{+} \backslash P\right) \backslash C$ is a strong deformation retract of both $A^{+}$and $\Omega^{+} \backslash P$ via projection in the direction orthogonal to $H$, and similarly for $A^{-}$.

Since $A^{+}$and $A^{-}$are open sets, the pair $\left(A^{+}, A^{-}\right)$is an excisive couple and therefore has a Mayer-Vietoris long exact sequence of singular homology (see [Mu, p. 186]):

$$
\cdots \longrightarrow H_{i}\left(A^{+}\right) \oplus H_{i}\left(A^{-}\right) \longrightarrow H_{i}\left(A^{+} \cup A^{-}\right) \longrightarrow H_{i-1}\left(A^{+} \cap A^{-}\right) \longrightarrow \cdots .
$$

Exactness implies that

$$
\beta_{i}\left(A^{+} \cup A^{-}\right) \leq \beta_{i}\left(A^{+}\right)+\beta_{i}\left(A^{-}\right)+\beta_{i-1}\left(A^{+} \cap A^{-}\right) .
$$

Since $A^{+} \cup A^{-}=\Omega \backslash P$ and $A^{+} \cap A^{-}=C$, the homotopy equivalences (2) and (3) imply that (4) is equivalent to (1). 
The inequality

$$
\ell_{n-i}^{+}(T) \geq \tilde{\beta}_{i-1}(\Omega \backslash P)
$$

can be proved by induction in a similar way. The basis for induction (the $|T|=1$ case) is easily checked. The argument for the induction step is, to begin with, identical to the preceding one, reducing the problem to proving the reduced Betti number inequality

$$
\tilde{\beta}_{i}(\Omega \backslash P) \leq \tilde{\beta}_{i}\left(\Omega^{+} \backslash P\right)+\tilde{\beta}_{i}\left(\Omega^{-} \backslash P\right)+\tilde{\beta}_{i-1}\left(\Omega^{0} \backslash P\right) .
$$

If $\Omega^{0} \backslash P \neq \varnothing$ we can proceed as before, this time however using the MayerVietoris sequence of reduced singular homology [Mu, p. 187]. If $\Omega^{0} \backslash P=\varnothing$ then $\left(1^{\prime}\right)$ is in fact an equality for all $i$, since $\Omega \backslash P$ is the union of disjoint open sets $\Omega^{+} \backslash P$ and $\Omega^{-} \backslash P$ and $\tilde{\beta}_{-1}(\varnothing)=1$.

Corollary 2.3. For any closed polyhedron $P \subseteq \mathbb{R}^{n}$,

$$
\begin{aligned}
& \ell^{-}(P) \geq \beta\left(\mathbb{R}^{n} \backslash P\right)=\sum_{i=0}^{n} \beta_{i}\left(\mathbb{R}^{n} \backslash P\right), \\
& \ell^{+}(P) \geq \beta\left(\mathbb{R}^{n} \backslash P\right)-1 .
\end{aligned}
$$

The weaker inequality $\ell^{-}(P) \geq \beta_{0}\left(\mathbb{R}^{n} \backslash P\right)$ has been known since the work of Dobkin and Lipton [DL]. It is the basis of the "component count method", which was later extended to algebraic decision trees by Steele and Yao [SY] and Ben-Or [BO].

The following alternative version of Corollary 2.3 shows that it is in fact a strengthening of Theorem 2.1, since $\chi(\hat{P})=\sum(-1)^{i} \beta_{i}(\hat{P})$. Applying Alexander duality in $S^{n} \cong \widehat{\mathbb{R}^{n}}$ (see [Mu, p. 424]), one gets

Corollary 2.4. For any closed polyhedron $P \subseteq \mathbb{R}^{n}$,

$$
\begin{aligned}
& \ell^{-}(P) \geq \beta(\hat{P})=\sum_{i=0}^{n} \beta_{i}(\hat{P}), \\
& \ell^{+}(P) \geq \beta(\hat{P})-1 .
\end{aligned}
$$

To illustrate the improvement of Corollary 2.4 over Theorem 2.1, let us consider the $k$-equal problem in $\mathbb{R}^{n}$ (see $\S 6 \mathrm{a}$ ). The Betti numbers of $M_{n, k}=\mathbb{R}^{n} \backslash P$ for the closed polyhedron $P=V_{\mathscr{A}_{n, k}}$ in question have been computed in [BW]. For instance, the Betti numbers of $M_{7,3}$ are $(1,351,350,0,0,0,0,0)$. The 3-equal problem is particularly interesting from this point of view, since $\chi\left(M_{n, 3}\right)=0$ whenever $n \equiv 3(\bmod 4)$, as can be seen from (6.1). Hence, in these cases the Euler characteristic lower bound gives nothing, while the Betti number bound guarantees at least $702(\approx 6$ !) NO-leaves for $n=7$, over 2 million $(\approx 6 \cdot 9$ !) NO-leaves for $n=11$, and over 23 billion $(\approx 4 \cdot 13$ !) NOleaves for $n=15$. These numbers for the 3-equal problem should be compared with the number of NO-leaves which the component count method gives for the 2-equal problem, which is $n !$. 
It should be remarked that there is no upper bound on $\ell^{-}(P)$ in terms of $\beta(\hat{P})$, or in terms of any topological invariant of $P$. If $P$ is a full-dimensional convex polytope in $\mathbb{R}^{n}$ then it is clear that points $x \in \mathbb{R}^{n} \backslash P$ sufficiently near the centers of gravity of different facets must go to different NO-leaves in any linear decision tree, and hence $\ell^{-}(P)$ equals at least the number of facets of $P$. On the other hand, $\beta(\hat{P})=2$ and all convex full-dimensional polytopes are topologically the same.

We leave open the question whether Corollary 2.3 allows a generalization to algebraic decision trees, that is, trees $T$ where the sign of a polynomial $q(x)$ of the variables determines the branching at each node. The weaker bounds in Theorem 2.1 were generalized to algebraic decision trees by Yao [Y].

Of course, Corollary 2.3 does not remain valid directly as stated for algebraic decision trees. For example, membership in any real algebraic set can be tested by an algebraic decision tree with a single node, while of course it can have very large Betti numbers. We suggest the following as a plausible generalization:

Conjecture 2.5. Let $P \subseteq \mathbb{R}^{n}$ be a closed semialgebraic set and $T$ an algebraic decision tree for $P$. Let $L^{-}$be the set of NO-leaves of $T$. Then

$$
\sum_{w \in L^{-}} \beta\left(P_{w}\right) \geq \beta\left(\mathbb{R}^{n} \backslash P\right)
$$

In the linear case $\beta\left(P_{w}\right)=1$ for all $w$, so Corollary 2.3 would follow. To obtain bounds on the depth of $T$ in the nonlinear case, one could stipulate that all test-polynomials have degree bounded by $d$, and then use the OleinikPetrovsky-Milnor-Thom Theorem [OP, O, Mi, Th] just as in Yao [Y].

Remark 2.6. Another possibility to use topology in estimating $\ell^{-}$is to blow up each $P_{w}\left(w \in L^{-}\right)$of dimension less than $n$ to an open convex set $U_{w}$ disjoint from $P$ (this is easy to do), and then apply the nerve theorem to show that $\mathbb{R}^{n} \backslash P$ is homotopy equivalent to the nerve of the family $\left\{U_{w}\right\}$, i.e., to a simplicial complex on $\ell^{-}$vertices. However, at least in the $k$-equal problem, the known bounds [BK] on the number of vertices of a simplicial complex with given Betti numbers do not seem to be strong enough.

\section{ARRANGEMENTS OF CONVEX SETS AND OF SUBSPACES}

For any finite family $\mathscr{A}=\left\{K_{1}, \ldots, K_{m}\right\}$ of sets in $\mathbb{R}^{n}$, let

$$
V_{\mathscr{A}}=\bigcup_{i=1}^{m} K_{i} \quad \text { and } \quad M_{\mathscr{A}}=\mathbb{R}^{n} \backslash V_{\mathscr{A}}
$$

We will always assume that no $K_{i}$ contains another $K_{j}$. If all sets $K_{i}$ are convex polyhedra (e.g., affine subspaces) then $V_{\mathscr{A}}$ and $M_{\mathscr{A}}$ are polyhedra. Recall that we denote by $\hat{V}_{\mathscr{A}}$ the compactification of $V_{\mathscr{A}}$ by a single point "at infinity".

In this section we derive topological and combinatorial bounds on the size of a linear decision tree $T$ testing membership in a polyhedron $P$, presented 
as the union $V_{\mathscr{A}}$ of an arrangement $\mathscr{A}$ of closed convex polyhedra. By the general results in the previous section we have

$$
\ell^{-}\left(V_{\mathscr{A}}\right) \geq \beta\left(\hat{V}_{\mathscr{A}}\right)=\beta\left(M_{\mathscr{A}}\right) \geq\left|\chi\left(\hat{V}_{\mathscr{A}}\right)\right|,\left|\chi\left(M_{\mathscr{A}}\right)\right|,
$$

and

$$
\ell^{+}\left(V_{\mathscr{A}}\right) \geq \tilde{\beta}\left(\hat{V}_{\mathscr{A}}\right)=\tilde{\beta}\left(M_{\mathscr{A}}\right) \geq\left|\chi\left(\hat{V}_{\mathscr{A}}\right)-1\right|=\left|\chi\left(M_{\mathscr{A}}\right)-1\right| .
$$

The problem lies in computing, or estimating, the topological invariants on the right-hand side. It turns out that much of this information is contained in the following combinatorial structure.

For any family $\mathscr{A}=\left\{K_{1}, \ldots, K_{m}\right\}$ of sets in $\mathbb{R}^{n}$, we define the intersection semilattice $L_{\mathscr{A}}$ of $\mathscr{A}$ as the partially ordered set of all nonempty intersections $K_{i_{1}} \cap \cdots \cap K_{i_{j}}: 1 \leq i_{1}<\cdots<i_{j} \leq m$, ordered by reverse inclusion. This semilattice has least element $\hat{0}=\mathbb{R}^{n}$. In case $\cap \mathscr{A}=K_{1} \cap \cdots \cap K_{m} \neq \varnothing$ there is also a greatest element $\hat{\mathbf{1}}=\bigcap \mathscr{A}$, and we may speak of the intersection lattice $L_{\mathscr{A}}$. The atoms of $L_{\mathscr{A}}$ (elements covering $\hat{0}$ ) are the sets $K_{i}$.

For the cases we are interested in some very useful aspects of the topology of the spaces $V_{\mathscr{A}}$ and $M_{\mathscr{A}}$ are encoded into the lattice $L_{\mathscr{A}}$, as the following results show.

The order complex $\Delta(P)$ of a poset $P$ is the simplicial complex whose vertex set is $P$ and whose simplices are the chains $x_{1}<\cdots<x_{k}$ in $P$. Note that if the elements of $P$ are vertices of a $(|P|-1)$-dimensional simplex, and each chain is represented by the convex hull of its elements, then we get a convex cell complex. The union of these simplices is a polyhedron (the geometric realization), which we shall also denote by $\Delta(P)$.

Proposition 3.1. Let $\mathscr{A}$ be a family of closed convex sets in $\mathbb{R}^{n}$. Then $V_{\mathscr{A}}$ and $\Delta\left(L_{\mathscr{A}} \backslash\{\hat{0}\}\right)$ are of the same homotopy type.

Proof. The covering of $V_{\mathscr{A}}$ by the closed subsets $K_{1}, \ldots, K_{m}$ and the covering of $\Delta\left(L_{\mathscr{A}} \backslash\{\hat{0}\}\right)$ by the subcomplexes $\Delta\left(\left\{x \in L_{\mathscr{A}}: x \geq K_{i}\right\}\right), i=1, \ldots, m$, have the same nerve. In both cases all nonempty intersections are contractible. Hence the result follows from the nerve theorem (see [B1, (10.7)]).

If $P$ is a bounded polyhedron, represented as the union of a family $\mathscr{A}$ of convex polytopes, then Proposition $3.1 \mathrm{can}$ be used to translate the topological bounds given in $\S 2$ to a more combinatorial form.

Unfortunately, if $P$ is unbounded the situation gets more complicated, since it is the topology of $\hat{V}_{\mathscr{A}}$, and not of $V_{\mathscr{A}}$, that the results of $\S 2$ involve. So Proposition 3.1 cannot be directly applied. In [BLY] the following trick was used: let us intersect $V_{\mathscr{A}}$ with a large simplex $Q$. This makes the membership problem only slightly more difficult: first test membership in $Q$; this can be done with a linear decision tree with $2^{n+1}-1$ YES-leaves corresponding to $Q$ 's open faces; then test membership in $V_{\mathscr{A}}$. Now, $Q \cap V_{\mathscr{A}}$ is a bounded polyhedron, and Proposition 3.1 can be applied to show that it is homotopy equivalent to the order complex $\Delta\left(L_{\mathscr{A}} \backslash\{\hat{0}\}\right)$ (which then can be handled by combinatorial tools, as we shall later see). Thus Theorem 2.1 gives 
Proposition 3.2. Let $\mathscr{A}$ be a family of closed convex polyhedra in $\mathbb{R}^{n}$.

(a) If $V_{\mathscr{A}}$ is bounded, then

$$
\ell^{+}\left(V_{\mathscr{A}}\right) \geq\left|\chi\left(\Delta\left(L_{\mathscr{A}} \backslash\{\hat{0}\}\right)\right)\right| .
$$

(b) If $V_{\mathscr{A}}$ is unbounded, then

$$
\ell^{+}\left(V_{\mathscr{A}}\right) \geq 2^{-n-1}\left|\chi\left(\Delta\left(L_{\mathscr{A}} \backslash\{\hat{0}\}\right)\right)\right| \text {. }
$$

If $\cap \mathscr{A} \neq \varnothing$, then $V_{\mathscr{A}}, Q \cap V_{\mathscr{A}}$, and $\Delta\left(L_{\mathscr{A}} \backslash\{\hat{0}\}\right)$ are contractible, so their topology is trivial and no information about $\ell^{+}\left(V_{\mathscr{A}}\right)$ is obtained. In this situation [BLY] uses another trick to obtain nontrivial bounds: the polyhedron $V_{\mathscr{A}}$ is intersected by a hyperplane $H$, disjoint from $\bigcap \mathscr{A}$, but meeting every other subset arising as the intersection of members of $\mathscr{A}$. Such a hyperplane can be found, e.g., if all polyhedra in $\mathscr{A}$ are affine subspaces. In that case any linear decision tree for $V_{\mathscr{A}}$ yields (by restricting the inputs) a linear decision tree for $V_{\mathscr{A}} \cap H$ with the same number of YES- and NO-leaves. The intersection lattice of $V_{\mathscr{A}} \cap H$ is isomorphic to $L_{\mathscr{A}} \backslash\{\hat{1}\}$, so the following bounds can be deduced [BLY, Equation (4.2)]:

$$
\ell^{+}\left(V_{\mathscr{A}}\right) \geq 2^{-n-1}\left|\chi\left(\Delta\left(L_{\mathscr{A}} \backslash\{\hat{0}, \hat{1}\}\right)\right)\right| .
$$

The idea to intersect with a generic hyperplane can be repeated; the effect on the intersection lattice of each generic hyperplane section is to remove the subspaces of currently minimal dimension. Combinatorially this corresponds to upper truncations of the intersection semilattice. Let

$$
L_{\mathscr{A}}^{p-t r u n c}=L_{\mathscr{A}} \backslash\left\{x \in L_{\mathscr{A}}: \operatorname{dim}(x)<p\right\} .
$$

Then this argument leads to the following generalization of (3.1).

Proposition 3.3. Let $\mathscr{A}$ be a family of affine subspaces in $\mathbb{R}^{n}$. Then for all $p \geq 0$,

$$
\ell^{+}\left(V_{\mathscr{Q}}\right) \geq 2^{-n-1}\left|\chi\left(\Delta\left(L_{\mathscr{A}}^{p-\operatorname{trunc}} \backslash\{\hat{0}\}\right)\right)\right| .
$$

Similar bounds can be derived for $\ell^{-}\left(V_{\mathscr{A}}\right)$. The tricks that were used to deal with the unbounded case weaken the lower bounds on the decision tree complexity; the loss is marginal in terms of the depth but more substantial in terms of the size.

To improve our estimates it would clearly be desirable to have a version of Proposition 3.1 describing the homotopy type (or homology) of $\hat{V}_{\mathscr{A}}$ rather than the homotopy type of $V_{\mathscr{A}}$. For the important case of subspace arrangements there is a result of Goresky and MacPherson [GM], which expresses the cohomology of $M_{\mathscr{A}}$ (and so, by Alexander duality, also the homology of $\hat{V}_{\mathscr{A}}$ ) in terms of the intersection semilattice $L_{\mathscr{A}}$. There is also a strengthening due to Ziegler and Živaljević [ZŽ] which expresses the homotopy type of $\hat{V}_{\mathscr{A}}$ in terms of the semilattice $L_{\mathscr{A}}$, but since our bounds depend only on Betti numbers it suffices to use the cohomology formula.

By an affine subspace arrangement we mean a finite family $\mathscr{A}=\left\{K_{1}, \ldots, K_{m}\right\}$ of affine subspaces in $\mathbb{R}^{n}$. Such an arrangement is called central if each $K_{i}$ is 
a linear subspace, i.e., it contains 0 . Every affine subspace is clearly a closed convex polyhedron. Subspace arrangements and their intersection lattices $L_{\mathscr{A}}$ have some special structure not shared by the more general families $\mathscr{A}$ of convex sets considered so far. The survey [B2] gives some general information about subspace arrangements.

Let $\tilde{H}^{i}$ denote reduced singular cohomology groups, and $\tilde{H}_{i}$ denote reduced simplicial homology groups, in both cases with coefficients in $\mathbb{Z}$. (Recall that in reduced homology, $\tilde{H}_{-1}(\varnothing) \cong \mathbb{Z}$ and $\tilde{H}_{i}(\varnothing)=0$ for all $i \neq-1$, while rank $\tilde{H}_{0}(X)$ is one less than the number of connected components, for nonempty spaces $X$.) Also, let $\tilde{\beta}_{i}$ denote reduced Betti numbers, i.e., the ranks of the reduced (co)homology groups. Further, let $\Delta(\hat{0}, x)$ be the order complex of the open interval $(\hat{0}, x)=\{z \mid \hat{0}<z<x\}$ in $L_{\mathscr{A}}$.

Proposition 3.4 (Goresky and MacPherson [GM, p. 238]). Let $\mathscr{A}$ be an affine subspace arrangement in $\mathbb{R}^{n}$. Then

$$
\tilde{H}^{i}\left(M_{\mathscr{A}}\right) \cong \bigoplus_{x>0, x \in L_{\mathscr{A}}} \tilde{H}_{n-\operatorname{dim}(x)-2-i}(\Delta(\hat{0}, x)) .
$$

The proof of Proposition 3.4 in [GM] uses methods from Morse theory. Other proofs are also known, e.g., via the homotopy type formula of Ziegler and $\check{Z}$ ivaljević $[Z \check{Z}]$.

The Goresky-MacPherson formula implies the following expressions for the reduced Betti numbers and the Euler characteristic of $M_{\mathscr{A}}$ (and via Alexander duality $\left.\hat{V}_{\mathscr{A}}\right)$.

Corollary 3.5. Let $\mathscr{A}$ be an affine subspace arrangement in $\mathbb{R}^{n}$. Then

$$
\tilde{\beta}_{i}\left(\hat{V}_{\mathscr{A}}\right)=\tilde{\beta}^{n-i-1}\left(M_{\mathscr{A}}\right)=\sum_{\substack{x \in L_{\mathscr{A}} \\ x>0}} \tilde{\beta}_{i-\operatorname{dim}(x)-1}(\Delta(\hat{0}, x)),
$$

and

$$
\chi\left(\hat{V}_{\mathscr{A}}\right)-1=(-1)^{n-1}\left(\chi\left(M_{\mathscr{A}}\right)-1\right)=\sum_{\substack{x \in L_{\mathscr{A}} \\ x>0}}(-1)^{\operatorname{dim}(x)-1}(\chi(\Delta(\hat{0}, x))-1) .
$$

The advantage of expressing the Betti numbers and the Euler characteristic of $\hat{V}_{\mathscr{A}}$ and $M_{\mathscr{A}}$ in terms of the Betti numbers and Euler characteristic of posets is that we can now invoke the powerful theory of Möbius functions (see [S3, Chapter 3] for an introduction to this theory). The key is the following theorem of $\mathrm{Ph}$. Hall [S3, p. 120].

Proposition 3.6. The value of the Möbius function $\mu(x, y)$ for a pair $x<y$ in a poset $P$ is one less than the Euler characteristic $\chi(\Delta(x, y))$ of the order complex of the open interval $(x, y)=\{z \in P \mid x<z<y\}$.

Consequently,

$$
\mu(x, y)=\sum_{i}(-1)^{i} \tilde{\beta}_{i}(x, y),
$$

where $\tilde{\beta}_{i}(x, y)$ denotes the $i$ th reduced Betti number of $\Delta(x, y)$. 
We can now summarize the lower bounds that have been obtained for the size of linear decision trees in terms of the Möbius function. For some of these formulas it is convenient to use the augmented intersection lattice $\hat{L}_{\mathscr{A}}=$ $L_{\mathscr{A}} \cup\{1\}$, obtained by creating a new element $\mathbb{1}=\varnothing$ greater than all elements of $L_{\mathscr{A}}$. (Note that $\hat{1}<1$ in $\hat{L}_{\mathscr{A}}$, when a top element $\hat{1}$ of $L_{\mathscr{A}}$ already exists.) For any semilattice $L$, we denote by $\mu_{L}$ its Möbius function.

Theorem 3.7. Let $\mathscr{A}=\left\{K_{1}, \ldots, K_{m}\right\}$ be a family of closed convex polyhedra in $\mathbb{R}^{n}$.

(a) If $V_{\mathscr{A}}$ is bounded, then

$$
\ell^{+}\left(V_{\mathscr{A}}\right) \geq\left|\mu_{\hat{L}_{\mathscr{A}}}(\hat{0}, \mathbf{1})+1\right| .
$$

(b) If $V_{\mathscr{A}}$ is unbounded, then

$$
\ell^{+}\left(V_{\mathscr{A}}\right) \geq 2^{-n-1}\left|\mu_{\hat{L}_{\mathscr{A}}}(\hat{0}, \mathbb{1})+1\right| .
$$

(c) If all polyhedra $K_{i}$ are affine subspaces, then

$$
\ell^{-}\left(V_{\mathscr{A}}\right) \geq 1+\sum_{\substack{x \in L_{\mathscr{\infty}} \\ x>0}} \sum_{i} \tilde{\beta}_{i}(\hat{0}, x) \geq \sum_{x \in L_{\mathscr{\alpha}}}\left|\mu_{L_{\mathscr{\alpha}}}(\hat{0}, x)\right| .
$$

In particular, in the central case

$$
\ell^{-}\left(V_{\mathscr{A}}\right) \geq\left|\mu_{L_{\mathscr{A}}}(\hat{0}, \hat{1})\right|,
$$

and in the noncentral case

$$
\ell^{-}\left(V_{\mathscr{A}}\right) \geq\left|\mu_{\hat{L}_{\mathscr{A}}}(\hat{0}, \mathbb{1})\right| .
$$

(d) If all polyhedra $K_{i}$ are affine subspaces, then

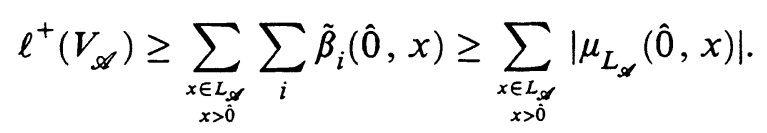

In particular, in the central case

$$
\ell^{+}\left(V_{\mathscr{A}}\right) \geq\left|\mu_{L_{\mathscr{A}}}(\hat{0}, \hat{1})\right|,
$$

and in the noncentral case

$$
\ell^{+}\left(V_{\mathscr{A}}\right) \geq\left|\mu_{\hat{L}_{\mathscr{A}}}(\hat{0}, \mathbb{1})+1\right| .
$$

Proof. These formulas combine information from 2.3, 3.2, 3.5, and 3.6. The last formulas in (c) and (d) follow in the noncentral case from $\mu_{\hat{L}_{\infty}}(\hat{0}, 1)=$ $-\sum_{x \in L_{\alpha}} \mu(\hat{0}, x)$.

Although the evaluation of the Möbius function is a difficult problem in general, its theory is fairly well developed and we shall illustrate the use of this theory in the next sections. 
The following is a restatement of the formula for $\chi\left(M_{\mathscr{A}}\right)$ in Corollary 3.5 in terms of the Möbius function. We will give an independent and more elementary proof in the central case, based on Proposition 3.1. (This proof can be extended from the central to the affine case, but since we will not make use of the noncentral case of the formula we omit this extension, which is a bit more involved.)

Corollary 3.8. For any subspace arrangement $\mathscr{A}$ in $\mathbb{R}^{n}$ :

$$
\chi\left(M_{\mathscr{A}}\right)=\sum_{x \in L_{\mathscr{A}}}(-1)^{n-\operatorname{dim}(x)} \mu(\hat{0}, x) .
$$

Proof. Suppose that $\mathscr{A}$ is central with intersection lattice $L=L_{\mathscr{A}}$, and let $V_{\mathscr{A}}^{o}=V_{\mathscr{A}} \cap S^{n-1}$ be the intersection with the unit sphere in $\mathbb{R}^{n}$. We may assume that $\cap \mathscr{A}=\{0\}$. Now, $M_{\mathscr{A}}$ deformation retracts radially onto $M_{\mathscr{A}} \cap S^{n-1}$, so via Alexander duality in $S^{n-1}$ the formula is equivalent to

$$
\chi\left(V_{\mathscr{A}}^{o}\right)=1+\sum_{\substack{x \in L \\ x>0}}(-1)^{\operatorname{dim}(x)} \mu(\hat{0}, x) .
$$

We will prove (3.3) by induction on $n$; the $\mathbb{R}^{2}$ case is easy to check.

For the induction step, let $H$ be a central hyperplane which is generic with respect to $\mathscr{A}$. Let $H^{+}$and $H^{-}$be the closed half-spaces determined by $H$. Then both $V_{\mathscr{A}}^{o} \cap H^{+}$and $V_{\mathscr{A}}^{o} \cap H^{-}$are homotopy equivalent to $\Delta(L \backslash\{\hat{0}, \hat{1}\})$. This follows from the nerve theorem by the same argument as in Proposition 3.1. Hence

$$
\chi\left(V_{\mathscr{A}}^{o} \cap H^{+}\right)-1=\chi\left(V_{\mathscr{A}}^{o} \cap H^{-}\right)-1=\mu_{L}(\hat{0}, \hat{1}) .
$$

On the other hand, $\mathscr{A}^{\prime}=\{H \cap K: K \in \mathscr{A}\}$ is a central arrangement in $H \cong \mathbb{R}^{n-1}$ whose intersection lattice $L^{\prime}$ is the upper truncation of $L$, i.e., $L^{\prime}=L \backslash\{x \in L: \operatorname{dim}(x)=1\}$. The dimension in $L^{\prime}$ of subspaces other than $\{0\}$ has been reduced by one. So the induction assumption gives

$$
\chi\left(V_{\mathscr{A}}^{o} \cap H\right)-1=\mu_{L^{\prime}}(\hat{0}, \hat{1})+\sum_{\substack{x \in L \\ 1<\operatorname{dim}(x)<n}}(-1)^{\operatorname{dim}(x)-1} \mu_{L}(\hat{0}, x) .
$$

Using the fact that the Euler characteristic is additive together with the defining recursion for the Möbius function, we get from these formulas:

$$
\begin{aligned}
\chi\left(V_{\mathscr{A}}^{o}\right) & =\chi\left(V_{\mathscr{A}}^{o} \cap H^{+}\right)+\chi\left(V_{\mathscr{A}}^{o} \cap H^{-}\right)-\chi\left(V_{\mathscr{Q}}^{o} \cap H\right) \\
& =1+2 \mu_{L}(\hat{0}, \hat{1})-\mu_{L^{\prime}}(\hat{0}, \hat{1})-\sum_{\substack{x \in L \\
1<\operatorname{dim}(x)<n}}(-1)^{\operatorname{dim}(x)-1} \mu_{L}(\hat{0}, x) \\
& =1+\mu_{L}(\hat{0}, \hat{1})-\sum_{\operatorname{dim}(x)=1} \mu_{L}(\hat{0}, x)+\sum_{\substack{x \in L \\
1<\operatorname{dim}(x)<n}}(-1)^{\operatorname{dim}(x)} \mu_{L}(\hat{0}, x) \\
& =1+\sum_{\substack{x \in L \\
x>\hat{0}}}(-1)^{\operatorname{dim}(x)} \mu_{L}(\hat{0}, x) . \quad
\end{aligned}
$$




\section{The MöbIUS fUNCTION OF PARTITIONS WITH RESTRICTED BLOCK SIZES}

The intersection lattices we will be interested in have combinatorial descriptions in terms of set partitions. In this and the next section we will derive expressions for the Möbius function of these partition lattices that will be needed for the complexity lower bounds in $\S 6$. The arguments here are purely combinatorial, and we will work in a setting that is more general than what the applications require.

Let $\Pi_{n}$ denote the lattice of partitions of the set $[n]=\{1,2, \ldots, n\}$ ordered by refinement, and $\mu$ its Möbius function. For $2 \leq k \leq n$ let $\Pi_{n, k}$ be the subposet of partitions with no block sizes in $\{2,3, \ldots, k-1\}$. In particular, $\Pi_{n, 2}=\Pi_{n}$. (Remark: $\Pi_{n, k}$ was denoted by $\Pi_{n, k-1}$ in [BLY].) We observe that $\Pi_{n, k}$ is itself a lattice, whose join-operation is the same as that of $\Pi_{n}$ and whose meet-operation is that of $\Pi_{n}$ (coarsest common refinement) followed by breaking all blocks of size less than $k$ into singletons. Let $\mu_{n, k}$ denote the Möbius function of $\Pi_{n, k}$. We set $\mu_{k}(n)=\mu_{n, k}(\hat{0}, \hat{1})$. We extend the definition to $1 \leq n<k$ by letting $\mu_{k}(1)=1$ and $\mu_{k}(2)=\cdots=\mu_{k}(k-1)=0$.

More generally, given any set $T \subseteq \mathbb{Z}_{+}$with $1 \in T$, we consider the set $\Pi_{n, T}$ of partitions of $[n]$ into blocks whose sizes are in $T$. Ordering the elements of $\Pi_{n, T}$ by refinement we get a poset, which is not a lattice in general. However, the discrete partition $\hat{0}$ does belong to $\Pi_{n, T}$. We denote by $\mu_{n, T}$ the Möbius function of the poset $\Pi_{n, T}$, where the subscript $n$ will often be suppressed. We also set $\mu_{T}(n)=\mu_{n, T}(\hat{0}, \hat{1})$, if $n \in T$. It will be convenient to extend the definition of $\mu_{n, T}(\pi, \sigma)$ by setting it to 0 if either one of the partitions $\pi$ and $\sigma$ is not in $\Pi_{n, T}$. In particular, $\mu_{T}(n)=0$ if $n \notin T$.

We will derive various formulas for the Möbius function $\mu_{T}$; specialized to $\mu_{k}$ one of these formulas will give a good enough estimate to settle the " $k$ equal" linear decision tree problem for all values of $k$ up to a constant factor. Another formula yields an expression for the Euler characteristic of the subset of $\mathbb{R}^{n}$ of points with no $k$ coordinates equal.

Set, for every $\pi \in \Pi_{n}$,

$$
s_{T}(\pi)=\sum_{\sigma \leq \pi} \mu_{T}(\hat{0}, \sigma)
$$

Also set $s_{T}(n)=s_{T}(\hat{1})$, and let $s_{T}(0)=1$. More generally, we shall consider the "characteristic polynomial" of $\Pi_{n, T}$, i.e., the polynomial

$$
\phi_{T}(n ; t)=\sum_{\pi \in \Pi_{n}} \mu_{T}(\hat{0}, \pi) t^{b(\pi)},
$$

where $b(\pi)$ denotes the number of blocks of $\pi$. Also let $\phi_{T}(0 ; t)=1$. Note that for $n>0$ we have

$$
\phi_{T}(n ; 0)=0, \quad \frac{d}{d t} \phi_{T}(n ; 0)=\mu_{T}(n), \quad \text { and } \quad \phi_{T}(n ; 1)=s_{T}(n) .
$$

The value of $\phi_{T}(n ;-1)$ will also concern us and will be of interest in the applications. (Note that in the definition of $\phi_{T}(n ; t)$ the summation could be set to run over all $\pi \in \Pi_{n, T}$ with the same result.) 
By the definition of the Möbius function, we have $s_{T}(\pi)=0$ for every $\pi \in \Pi_{n, T}, \pi \neq \hat{0}$. In particular, $s_{T}(n)=0$ if $n \in T \backslash\{1\}$. To extend this observation slightly, define $T^{*}=\mathbb{Z}_{+} \backslash(T \backslash\{1\})$.

Proposition 4.1. If $\pi \notin \Pi_{n, T^{*}}$ then $s_{T}(\pi)=0$.

Proof. Let $\pi=\left\{B_{1}, \ldots, B_{r}\right\} \in \Pi_{n}$. For any subset $B \subseteq[n]$, let $(B)$ denote the partition of $[n]$ where $B$ is one block and the rest are singletons. Then the poset $[\hat{0}, \pi] \cap \Pi_{n, T}$ is the direct product of the intervals $\left[\hat{0},\left(B_{i}\right)\right] \cap \Pi_{n, T}$. Hence

$$
\begin{aligned}
s_{T}(\pi) & =\sum_{\sigma \leq \pi} \mu_{n, T}(\hat{0}, \sigma)=\sum_{\sigma \leq \pi} \mu_{n, T}\left(\hat{0}, \sigma \wedge\left(B_{1}\right)\right) \cdots \mu_{n, T}\left(\hat{0}, \sigma \wedge\left(B_{r}\right)\right) \\
& =\sum_{\sigma_{1} \leq\left(B_{1}\right)} \cdots \sum_{\sigma_{r} \leq\left(B_{r}\right)} \mu_{n, T}\left(\hat{0}, \sigma_{1}\right) \cdots \mu_{n, T}\left(\hat{0}, \sigma_{r}\right)=s_{T}\left(\left|B_{1}\right|\right) \cdots s_{T}\left(\left|B_{r}\right|\right) .
\end{aligned}
$$

If one of the block sizes is in $T \backslash\{1\}$, then this product is 0 .

By Möbius inversion, we get the following formula for $\mu_{T}$.

Proposition 4.2. For every $\pi \in \Pi_{n}$,

$$
\mu_{T}(\hat{0}, \pi)=\sum_{\sigma \leq \pi} s_{T}(\sigma) \mu(\sigma, \pi) .
$$

Using the well-known formula

$$
\mu(\hat{0}, \hat{1})=(-1)^{n-1}(n-1) !,
$$

we get

$$
\mu_{T}(n)=\sum_{\sigma \in \Pi_{n, T^{*}}}(-1)^{b(\sigma)-1}(b(\sigma)-1) ! s_{T}(\sigma) .
$$

The characteristic polynomial satisfies the following useful recursion.

Proposition 4.3. $\phi_{T}(n ; t)=t \sum_{m=1}^{n}\left(\begin{array}{c}n-1 \\ m-1\end{array}\right) \mu_{T}(m) \phi_{T}(n-m ; t)$.

Proof. For $\pi \in \Pi_{n}$, let $\pi^{\prime}$ denote the partition obtained from $\pi$ by splitting all blocks not containing $n$ into singletons, and let $\pi^{\prime \prime}$ denote the partition obtained from $\pi$ by splitting the block containing $n$ into singletons. Recall also the definition of the partition $(B)$ from the proof of Proposition 4.1. Then the poset $[\hat{0}, \pi] \cap \Pi_{n, T}$ is the direct product of the posets $\left[\hat{0}, \pi^{\prime}\right] \cap \Pi_{n, T}$ and $\left[\hat{0}, \pi^{\prime \prime}\right] \cap \Pi_{n, T}$, and hence

$$
\mu_{T}(\hat{0}, \pi)=\mu_{T}\left(\hat{0}, \pi^{\prime}\right) \mu_{T}\left(\hat{0}, \pi^{\prime \prime}\right)
$$


This implies that

$$
\begin{aligned}
\phi_{T}(n ; t) & =\sum_{\pi} \mu_{T}(\hat{0}, \pi) t^{b(\pi)}=\sum_{\pi} \mu_{T}\left(\hat{0}, \pi^{\prime}\right) \mu_{T}\left(\hat{0}, \pi^{\prime \prime}\right) t^{b(\pi)} \\
& =\sum_{B \subseteq[n], n \in B} \mu_{T}(\hat{0},(B)) t \sum_{\pi: \pi^{\prime}=(B)} \mu_{T}\left(\hat{0}, \pi^{\prime \prime}\right) t^{b\left(\pi^{\prime \prime}\right)-|B|} \\
& =\sum_{B \subseteq[n], n \in B} \mu_{T}(\hat{0},(B)) t \phi_{T}(n-|B| ; t) \\
& =t \sum_{m=1}^{n}\left(\begin{array}{c}
n-1 \\
m-1
\end{array}\right) \mu_{T}(m) \phi_{T}(n-m ; t) .
\end{aligned}
$$

In particular, by putting $t=1$ we get the following recurrence for $s_{T}$ :

$$
s_{T}(n)=\sum_{m \leq n}\left(\begin{array}{c}
n-1 \\
m-1
\end{array}\right) \mu_{T}(m) s_{T}(n-m) .
$$

Let us introduce the exponential generating function

$$
G_{T}(x, t)=\sum_{n=0}^{\infty} \phi_{T}(n ; t) \frac{x^{n}}{n !},
$$

and its specializations

$$
\begin{aligned}
& F_{T}(x)=\sum_{n=1}^{\infty} \mu_{T}(n) \frac{x^{n}}{n !}=\left.\frac{\partial}{\partial t} G_{T}(x, t)\right|_{t=0}, \\
& p_{T}(x)=\sum_{n=0}^{\infty} s_{T}(n) \frac{x^{n}}{n !}=G_{T}(x, 1) .
\end{aligned}
$$

Proposition 4.3 is equivalent to the following differential equation:

$$
\frac{\partial}{\partial x} G_{T}(x, t)=t G_{T}(x, t) \frac{\partial}{\partial x} F_{T}(x) .
$$

Dividing by $G_{T}$, integrating, and checking the value at $x=0$, we obtain the following result.

Theorem 4.4. $G_{T}(x, t)=e^{t F_{T}(x)}$.

By this theorem, we can express $G_{T}$ and $F_{T}$ in terms of $p_{T}$ as follows:

\section{Corollary 4.5 .}

$$
\begin{aligned}
& F_{T}(x)=\ln p_{T}(x) \\
& G_{T}(x, t)=p_{T}(x)^{t}
\end{aligned}
$$

The first of these formulas has also been obtained by R. Stanley using a different method (personal communication).

In the case of finite $T^{*}$ we can extract a useful expression for the Möbius function $\mu_{T}$ from this functional equation. 
Theorem 4.6. Suppose that $T^{*}$ is finite and let $\alpha_{1}, \ldots, \alpha_{d}$ be the complex roots of the polynomial $p_{T}(x)=1+\sum_{n \in T^{*}} s_{T}(n) \frac{x^{n}}{n !}$. Then

$$
\mu_{T}(n)=-(n-1) ! \sum_{i=1}^{d} \alpha_{i}^{-n}
$$

Proof. The polynomial $p_{T}$ has constant term 1, so we can write

$$
p_{T}(x)=\prod_{i=1}^{d}\left(1-\frac{x}{\alpha_{i}}\right)
$$

Then

$$
F_{T}(x)=\sum_{i=1}^{d} \ln \left(1-\frac{x}{\alpha_{i}}\right)=-\sum_{i=1}^{d} \sum_{n=1}^{\infty} \frac{1}{n} \frac{x^{n}}{\alpha_{i}^{n}}=-\sum_{n=1}^{\infty} \frac{x^{n}}{n} \sum_{i=1}^{d} \alpha_{i}^{-n} .
$$

The result is obtained by comparing coefficients.

Clearly $d=\operatorname{deg} p_{T} \leq \max \left(T^{*}\right)$; however, equality does not hold in general as is shown in Remark 4.10 below.

In order to find a good expression for $\phi_{T}(n ;-1)$, we will assume that the polynomial $p_{T}$ has no multiple roots. Then

$$
G_{T}(x,-1)=\frac{1}{p_{T}(x)}=\frac{1}{\prod_{i=1}^{d}\left(1-x / \alpha_{i}\right)}=\sum_{i=1}^{d} \frac{B_{i}}{1-x / \alpha_{i}},
$$

where

$$
B_{i}=-\frac{1}{\alpha_{i} p_{T}^{\prime}\left(\alpha_{i}\right)}
$$

Thus

$$
G_{T}(x,-1)=-\sum_{i=1}^{d} \frac{1}{\alpha_{i} p_{T}^{\prime}\left(\alpha_{i}\right)} \frac{1}{1-x / \alpha_{i}}=-\sum_{i=1}^{d} \frac{1}{\alpha_{i} p_{T}^{\prime}\left(\alpha_{i}\right)} \sum_{n=0}^{\infty}\left(\frac{x}{\alpha_{i}}\right)^{n} .
$$

Comparing coefficients, we obtain

Theorem 4.7. If $T^{*}$ is finite and the complex roots $\alpha_{1}, \ldots, \alpha_{d}$ of $p_{T}$ are distinct, then

$$
\phi_{T}(n ;-1)=\sum_{\pi \in \Pi_{n, T}} \mu_{n, T}(\hat{0}, \pi)(-1)^{b(\pi)}=-n ! \sum_{i=1}^{d} \frac{1}{p_{T}^{\prime}\left(\alpha_{i}\right)} \alpha_{i}^{-n-1} .
$$

Let us now specialize the preceding discussion to the case of $\Pi_{n, k}$. Then $T^{*}=\{1, \ldots, k-1\}$, so we get from Proposition 4.1 that $s_{T}(\pi)=0$ unless every block in $\pi$ has size less than $k$. In particular, $s_{T}(n)=0$ for $n \geq k$. Moreover, $s_{T}(n)=1$ for $n<k$, since then the only nonzero term in the definition of $s_{T}(n)$ is for $\sigma=\hat{0}$. Consequently, in this case $s_{T}(n)$ is the characteristic function of $T^{*}$. 
Let $S_{k}(n, j)$ denote the number of partitions of [n] into $j$ parts, all of size less than $k$. Then formula (4.1) specializes to

$$
\mu_{k}(n)=\sum_{j=1}^{n}(-1)^{j-1}(j-1) ! S_{k}(n, j) .
$$

(Remark: In [BLY] this was derived as a special case of a result of Crapo.)

In the case of $\Pi_{n, k}$ we will denote $p_{T}$ by $p_{k}$. The description of $s_{T}(n)$ shows that this polynomial has a particularly nice form:

$$
p_{k}(x)=1+x+\frac{x^{2}}{2}+\cdots+\frac{x^{k-1}}{(k-1) !} .
$$

A lot of information about the distribution of the roots of the truncated exponential $p_{k}$ is available; sce, e.g., Szegö [Sz] and Varga [V, Chapter 4]. However, we shall make use only of some special facts which are easy to verify.

The formula for $\mu_{k}(n)$ in terms of the roots of $p_{k}$ that results as a special case of Theorem 4.6 was given in [BLY, Theorem 5.6]:

$$
\mu_{k}(n)=-(n-1) ! \sum_{i=1}^{k-1} \alpha_{i}^{-n}
$$

The polynomial $p_{3}(x)=1+x+\frac{x^{2}}{2}$ has roots $\alpha_{1}=-1+i$ and $\alpha_{2}=-1-i$. So from (4.3) we deduce that

$$
\mu_{3}(n)=0 \quad \text { if } n \equiv 2(\bmod 4)
$$

The roots of $p_{k}$ are distinct, since

$$
p_{k}^{\prime}\left(\alpha_{i}\right)=p_{k}\left(\alpha_{i}\right)-\frac{\alpha_{i}^{k-1}}{(k-1) !}=-\frac{\alpha_{i}^{k-1}}{(k-1) !} \neq 0 .
$$

Hence, from Theorem 4.7 we obtain

\section{Corollary 4.8 .}

$$
\phi_{k}(n ;-1)=\sum_{\pi \in \Pi_{n, k}} \mu_{n, k}(\hat{0}, \pi)(-1)^{b(\pi)}=n !(k-1) ! \sum_{i=1}^{k-1} \alpha_{i}^{-n-k}=-\frac{\mu_{k}(n+k)}{\left(\begin{array}{c}
n+k-1 \\
k-1
\end{array}\right)} \text {. }
$$

From (4.2) or (4.3) we get the following recurrence for $\mu_{k}$ :

$$
\mu_{k}(n)=-\sum_{r=1}^{k-1}\left(\begin{array}{c}
n-1 \\
r
\end{array}\right) \mu_{k}(n-r) .
$$

It is also worth mentioning that for $k \leq n \leq 2 k-1$, we have the following explicit formula:

$$
\mu_{k}(n)=(-1)^{n-k-1}\left(\begin{array}{l}
n-1 \\
k-1
\end{array}\right) \text {. }
$$

This follows either from (4.5) or from the observation that in this case $\Pi_{n, k}$ is a lower truncated Boolean algebra. Applying the recurrence, it is easy to go one step further and derive the formula

$$
\mu_{k}(2 k)=\left(\begin{array}{c}
2 k-1 \\
k-1
\end{array}\right)\left((-1)^{k-1}-1\right)
$$


In particular, $\mu_{k}(2 k)=0$ if $k$ is odd.

It follows from (4.5) that $\mu_{k}(n)$ is always divisible by $\left(\begin{array}{c}n-1 \\ k-1\end{array}\right)$. This is also a consequence of Corollary 4.8 and Proposition 4.9.

Formula (3.2) gives the expansion

$$
\mu_{k}(n)=\sum_{i}(-1)^{i} \tilde{\beta}_{i}\left(\Pi_{n, k}\right),
$$

in terms of the reduced Betti numbers $\widetilde{\beta}_{i}\left(\Pi_{n, k}\right)=\widetilde{\beta}_{i}(\hat{0}, \hat{1})$ of the order complex of $\Pi_{n, k} \backslash\{\hat{0}, \hat{1}\}, n \geq k$. These Betti numbers have been determined by Björner and Welker [BW] as follows.

Proposition 4.9. (a) $\tilde{\beta}_{i}\left(\Pi_{n, k}\right) \neq 0$ if and only if $i=n-3-t(k-2)$ for some integer $t$ such that $1 \leq t \leq\left\lfloor\frac{n}{k}\right\rfloor$.

(b) If $k>2$ and $1 \leq t \leq\left\lfloor\frac{n}{k}\right\rfloor$, then

$$
\begin{aligned}
\tilde{\beta}_{n-3-t(k-2)}\left(\Pi_{n, k}\right) & \\
=(t-1) !\left(\begin{array}{l}
n-1 \\
k-1
\end{array}\right) & \cdot \sum\left(\begin{array}{c}
n-k-1-i_{1} \\
k-1
\end{array}\right)\left(\begin{array}{c}
n-2 k-1-i_{2} \\
k-1
\end{array}\right) \\
\cdots & \left(\begin{array}{c}
n-(t-1) k-1-i_{t-1} \\
k-1
\end{array}\right) 1^{i_{1}-i_{0}} 2^{i_{2}-i_{1}} \cdots t^{i_{t}-i_{t-1}}
\end{aligned}
$$

with summation over all strings $0=i_{0} \leq i_{1} \leq \cdots \leq i_{t-1} \leq i_{t}=n-t k$.

We will end this section with a few remarks about general partition posets $\Pi_{n, T}$. More precisely, some interesting special choices for the set $T$ of allowed block sizes will be discussed.

Remark 4.10. Let $T_{1}=\mathbb{Z}_{+} \backslash\{2,6\}$ and $T_{0}=\mathbb{Z}_{+} \backslash\{2\}$. It follows from (4.4) that $s_{T_{1}}(6)=0$. Hence

$$
p_{T_{1}}(x)=1+x+\frac{x^{2}}{2}
$$

which has the surprising consequence (via Theorem 4.6) that

$$
\mu_{T_{1}}(n)=\mu_{T_{0}}(n) \text {, for all } n \text {. }
$$

This can by induction be extended to $T_{m}=\mathbb{Z}_{+} \backslash\{2,6,10, \ldots, 2+4 m\}$ :

$$
p_{T_{m}}(x)=1+x+\frac{x^{2}}{2} \quad \text { and } \quad \mu_{T_{m}}(n)=\mu_{T_{0}}(n),
$$

for all $m, n \geq 1$.

Remark 4.11. Let $T=\mathbb{Z}_{+} \backslash\{k\}$, for some $k \geq 2$. Then Corollary 4.5 gives

$$
\sum_{n \geq 1} \mu_{T}(n) \frac{x^{n}}{n !}=\ln \left(1+x+(-1)^{k} \frac{x^{k}}{k}\right) .
$$

Remark 4.12. Let $T=$ odd positive integers $\}$. This case has been studied by Calderbank, Hanlon, and Robinson [CHR]. On p. 291 of [CHR] two formulas 
are given which in our terminology say that

$$
\begin{aligned}
\mu_{T}(n) & =(-1)^{\frac{n-1}{2}}(1 \cdot 3 \cdots(n-2))^{2}, \quad \text { if } n \text { is odd } \\
s_{T}(n) & =(-1)^{\frac{n-2}{2}}(1 \cdot 3 \cdots(n-3))^{2}(n-1), \text { if } n \text { is even. }
\end{aligned}
$$

Insertion into the formula (Corollary 4.5)

$$
1+x+\sum_{n \text { even }} s_{T}(n) \frac{x^{n}}{n !}=\exp \left(\sum_{n \text { odd }} \mu_{T}(n) \frac{x^{n}}{n !}\right)
$$

gives rise to a curious identity.

Remark 4.13. Let $T=\{1, k\}$. Then

$$
\mu_{T}(n)= \begin{cases}1, & \text { if } n=1 \\ -1, & \text { if } n=k \\ 0, & \text { otherwise }\end{cases}
$$

and $s_{T}(n)$ has the following combinatorial interpretation.

Let $\Gamma_{n, k}$ denote the simplicial complex whose vertices are the $k$-element subsets of $[n]$ and whose simplices are collections of pairwise disjoint $k$-subsets. For $k=2$ the simplices are the matchings in the complete graph on $n$ nodes. Another description of $\Gamma_{n, k}$ in graph-theoretic terms is as the clique complex of the Kneser graph $K G_{n, k}$. Clearly,

$$
s_{T}(n)=-\tilde{\chi}\left(\Gamma_{n, k}\right)
$$

where $\tilde{\chi}$ denotes the reduced Euler characteristic.

Hence, Theorem 4.4 specializes to the following exponential generating function

$$
\sum_{n=0}^{\infty} \tilde{\chi}\left(\Gamma_{n, k}\right) \frac{x^{n}}{n !}=-e^{x-\frac{x^{k}}{k !}}
$$

The complexes $\Gamma_{n, k}$ have been studied in [BLVŽ], where it was shown that their reduced Betti numbers vanish in dimensions $\leq \frac{n-2 k-1}{2 k-1}$.

\section{THE MÖBIUS FUNCTION OF PARTITIONS WITH RESTRICTED NUMBER OF BLOCKS}

In this section we will discuss a subposet of $\Pi_{n}$ which is in many respects simpler to analyze than $\Pi_{n, k}$, namely,

$$
\Pi_{n}^{k}=\left\{\pi \in \Pi_{n}: b(\pi) \leq k\right\} \cup\{\hat{0}\},
$$

for $k \geq 1$. Here $b(\pi)$ denotes the number of blocks of the partition $\pi$. This can be described as a lower truncation of the partition lattice $\Pi_{n} ; n-1-k$ rank levels at the bottom have been removed if $k<n-1 ; \Pi_{n}^{k}=\Pi_{n}$ if $k \geq n-1$.

We let $\mu_{n}^{k}$ denote the Möbius function of this poset, and set in particular $\mu^{k}(n)=\mu_{n}^{k}(\hat{0}, \hat{1})$. Clearly $\mu^{k}(n)=(-1)^{n-1}(n-1)$ ! if $k \geq n-1$. 
Assume that $n>k$. Then by definition, we have

$$
\mu^{k}(n)=\mu^{k}(\hat{0}, \hat{1})=-\sum_{\pi>\hat{0}} \mu^{k}(\pi, \hat{1})=-\sum_{r=1}^{k} S(n, r)(-1)^{r-1}(r-1) !,
$$

where $S(n, r)$ is the number of partitions of an $n$-set into $r$ blocks (the Stirling numbers of the second kind). To bring this expression to a simpler form, we substitute the standard recursion for Stirling numbers:

$$
S(n, r)=S(n-1, r-1)+r S(n-1, r) .
$$

Then all terms in the middle cancel, and we get

Proposition 5.1. For $n>k$, we have

$$
\mu^{k}(n)=(-1)^{k} k ! S(n-1, k) .
$$

From this it is easy to determine the exponential generating function of $\mu^{k}$.

Corollary 5.2.

$$
\sum_{n=k}^{\infty} \mu^{k}(n+1) \frac{x^{n}}{n !}=\left(1-e^{x}\right)^{k}
$$

We will also derive a formula for the characteristic polynomial of $\Pi_{n}^{k}$. We define this polynomial by

$$
\phi^{k}(n ; t)=\sum_{\pi \in \Pi_{n}^{k}} \mu_{n}^{k}(\hat{0}, \pi) t^{b(\pi)} .
$$

Using the well-known formula

$$
\sum_{\pi \in \Pi_{n}} \mu(\hat{0}, \pi) t^{b(\pi)}=t(t-1) \cdots(t-n+1)
$$

[S3, p. 128], we have

$$
\begin{aligned}
\phi^{k}(n ; t) & =t^{n}+\sum_{b(\pi) \leq k} \mu^{k}(\hat{0}, \pi) t^{b(\pi)}=t^{n}-\sum_{b(\pi) \leq k} \sum_{\substack{\sigma \leq \pi \\
b(\sigma) \leq k}} \mu(\sigma, \pi) t^{b(\pi)} \\
& =t^{n}-\sum_{b(\sigma) \leq k} t(t-1) \cdots(t-b(\sigma)+1)=t^{n}-\sum_{r=1}^{k} S(n, r) r !\left(\begin{array}{l}
t \\
r
\end{array}\right) .
\end{aligned}
$$

In particular, we have

$$
\phi^{k}(n ;-1)=(-1)^{n}-\sum_{r=1}^{k}(-1)^{r} S(n, r) r ! .
$$

To bring this to a more useful nonalternating form, we substitute from (5.1), 
to get

$$
\begin{aligned}
\sum_{r=1}^{k}(-1)^{r} S(n, r) r !=\sum_{r=1}^{k}(-1)^{r}(S(n-1, r-1)+r S(n-1, r)) r ! \\
\quad=\sum_{r=1}^{k-1} S(n-1, r)\left((-1)^{r} r ! r+(-1)^{r+1}(r+1) !\right)+(-1)^{k} k ! k S(n-1, k) \\
\quad=\sum_{r=1}^{k-1} S(n-1, r)(-1)^{r+1} r !+(-1)^{k} k ! k S(n-1, k) .
\end{aligned}
$$

Repeating these transformations, we obtain the identity

$$
\sum_{r=1}^{k} S(n, r)(-1)^{r} r !=(-1)^{k} \sum_{r=1}^{k} r ! r S(n-k+r-1, r) .
$$

Thus we have proved the following result.

Theorem 5.3.

$$
\phi^{k}(n ;-1)=(-1)^{n}+(-1)^{k+1} \sum_{r=1}^{k} r ! r S(n-k+r-1, r) .
$$

\section{ApPlications}

A main motivation for this paper is to give lower bounds for the size of linear decision trees for certain specific problems. Using the general tools developed in earlier sections together with the Möbius function computations in Sections 4 and 5 we can now deal with these problems.

6a. The $k$-equal problem. "Given real numbers $x_{1}, x_{2}, \ldots, x_{n}$, decide whether $k$ of them are equal."

The following algorithm shows that the $k$-equal problem can be solved using $8 n \log _{3}(n / k)$ comparisons “ $x<y$ ?". Assume (for simplicity) that $n=2^{m} k$. We start with determining the $\left(2^{m-1} k\right)$ th largest element; this takes $3 n$ comparisons (see $[R])$. Then we go on with finding $\left(2^{m-2} k\right)$ th largest elements among those smaller and also among those larger than this element (ties are broken arbitrarily). In the $j$ th phase, those elements found so far split all elements into blocks of size $2^{m-j} k$, and we find the element of each block which splits it into two equal parts (where each element is counted in the block immediately before it).

After $m$ phases, we have found the $k$ th, $(2 k)$ th, $\ldots, 2^{m} k$ th largest elements. Now if there are $k$ equal elements, then one of these special elements must occur among them; therefore it is enough to compare each of them with $2 k$ other elements (in the blocks immediately before and after them) to see if indeed this is the case.

Each phase takes at most $3 n$ comparisons, so the total number of comparisons needed is at most $3 n m+(n / k) \cdot 2 k \leq 3 n \log _{2}(n / k)+2 n \leq 8 n \log _{3}(n / k)$. 
This determines a linear decision tree with depth at most $8 n \log _{3}(n / k)$ and (consequently) of size at most $(n / k)^{8 n}$.

For $k$ very close to $n(k>n / 2)$, we can do a little better. We find the median of the first $2(n-k)+1$ elements in at most $6(n-k)$ comparisons, and then compare this element with the rest. This gives a decision tree of depth $n+6(n-k)$. The size of this tree is at most $\left(\begin{array}{l}n \\ k\end{array}\right) 2^{11(n-k)}$. In fact, two YES-inputs lead to the same leaf, provided they lead to the same leaf of the median-finding subtree on the top, the positions of the first $k$ occurrences of the majority element are the same, and the nonmajority elements compare with the majority element the same way. Hence there are at most $\left(\begin{array}{l}n \\ k\end{array}\right) 3^{6(n-k)} 2^{n-k-1}<\left(\begin{array}{l}n \\ k\end{array}\right) 2^{11(n-k)-1}$ YES-leaves; a similar argument shows that there are at most $\left(\begin{array}{l}n \\ k\end{array}\right) 2^{11(n-k)-1}$ NOleaves. Note that this number is bounded by a polynomial in $n$ if $n-k$ is a constant.

The next result shows that this depth is best possible up to a constant, and this size is best possible up to a constant in the exponent.

Theorem 6.1. Every linear decision tree for the k-equal problem has size at least

$$
\max \left\{\left(\begin{array}{l}
n \\
k
\end{array}\right),(n / 3 k)^{n}\right\}
$$

and depth at least

$$
\max \left\{n-1, n \log _{3}(n / 3 k)\right\} .
$$

We start with some preliminary observations. The $k$-equal problem is a special case of the problem of testing membership in the union of a subspace arrangement. Let $\mathscr{A}_{n, k}$ denote the arrangement in $\mathbb{R}^{n}$ of the $\left(\begin{array}{l}n \\ k\end{array}\right)$ linear subspaces of dimension $n-k+1$ given by the equations $x_{i_{1}}=x_{i_{2}}=\cdots=x_{i_{k}}$, for $1 \leq i_{1}<\cdots<i_{k} \leq n$. It is clear that the $k$-equal problem is to decide whether $x \in V_{\mathscr{A}_{n, k}}$ for points $x \in \mathbb{R}^{n}$.

Let $\ell_{k}^{-}(n)\left[\ell_{k}^{+}(n)\right]$ denote the minimum number of NO-leaves [YES-leaves] in any linear decision tree for the $k$-equal problem. Both functions are monotone in $n$. In fact, let $m \leq n$. Every linear decision tree for $n$ elements can be used to obtain a linear decision tree for $m$ elements, by adding $n-m$ new elements $x, x^{2}, \ldots, x^{n-m}$, where $x$ is considered very large. The sign of a linear function of these $n$ elements is trivially determined unless all the new elements have coefficient 0 , in which case it is a linear decision about the old elements. The linear decision tree obtained this way has at most as many YES-leaves and NO-leaves as the original one.

By Theorem 3.7, the Möbius function of the corresponding intersection lattice will provide lower bounds for the size of decision trees. Such intersection lattices are in fact isomorphic to the block-size-restricted partition lattices $\Pi_{n, k}$ dealt with in $\S 4$.

Proposition 6.2. The intersection lattice of $\mathscr{A}_{n, k}, 2 \leq k \leq n$, is isomorphic to the lattice $\Pi_{n, k}$. If under this isomorphism a subspace $x \in \mathscr{A}_{n, k}$ corresponds to a partition in $\Pi_{n, k}$ with $j$ blocks, then $\operatorname{dim}(x)=j$. 
Proof. Associate with each partition $\pi \in \Pi_{n}$ the subspace

$$
B_{\pi}=\left\{x \in \mathbb{R}^{n}: x_{i}=x_{j} \text { whenever } i \text { and } j \text { belong to the same block of } \pi\right\} \text {. }
$$

Clearly, $\operatorname{dim}\left(B_{\pi}\right)=b(\pi)$, and $B_{\pi} \cap B_{\sigma}=B_{\pi \vee \sigma}$. So the identification of the subspace $x_{i_{1}}=x_{i_{2}}=\cdots=x_{i_{k}}$ with the partition whose only nontrivial block is $\left\{i_{1}, i_{2}, \ldots, i_{k}\right\}$ extends to an identification of the intersection lattice of $\mathscr{A}_{n, k}$ with $\Pi_{n, k}$.

From this and Corollaries 3.8 and 4.8 we get

\section{Theorem 6.3.}

$$
\chi\left(M_{\mathscr{A}_{n, k}}\right)=(-1)^{n} n !(k-1) ! \sum_{i=1}^{k-1} \alpha_{i}^{-n-k}=(-1)^{n-1} \frac{\mu_{k}(n+k)}{\left(\begin{array}{c}
n+k-1 \\
k-1
\end{array}\right)},
$$

where $\alpha_{1}, \ldots, \alpha_{k-1}$ are the roots of $\sum_{i<k} \frac{x^{i}}{i !}$.

The periodic vanishing of $\mu_{3}(n)$ mentioned in (4.4) implies that

$$
\chi\left(M_{\mathscr{A}_{n, 3}}\right)=0 \quad \text { when } n \equiv 3(\bmod 4) \text {. }
$$

The Betti numbers of $M_{\mathscr{A}_{n, k}}$ were determined in [BW], however not in a form that allows a simple closed formula. Nonzero Betti numbers occur only in dimensions that are multiples of $k-2$; therefore Euler characteristic and sum of Betti numbers give the same lower bound for the $k$-equal-problem when $k$ is even.

We now obtain from Theorems 2.1, 2.2, 3.7, and 6.3:

Corollary 6.4. Let $T$ be a linear decision tree for the $k$-equal problem for $n$ numbers. Then

(a) $\ell^{+}(T), \ell^{-}(T) \geq \frac{\left|\mu_{k}(n+k)\right|}{\left(\begin{array}{c}n+k-1 \\ k-1\end{array}\right)}$,

(b) $\ell^{+}(T), \ell^{-}(T) \geq\left|\mu_{k}(n)\right|$,

(c) $\ell^{+}(T), \ell^{-}(T) \geq \sum_{i} \tilde{\beta}_{i}\left(\Pi_{n, k}\right)$.

The lower bound (b) will now be used to give a self-contained proof of the asymptotic lower bound for the $k$-equal problem, based on the Möbius function computations in $\S 4$. Then we will use the sharper bound (c) and the expressions for the Betti numbers $\tilde{\beta}_{i}\left(\Pi_{n, k}\right)$ appearing in Proposition 4.9 to give a (slight) improvement. We have chosen to work out both of these two parallel approaches in order to illustrate the variety of techniques that our general lower bound method allows.

For the first approach we would like to show, using the formulas developed in $\S 4$, that $\left|\mu_{k}(n)\right|$ is large. Unfortunately, this is not always true; e.g., (4.4) shows that $\mu_{3}(n)=0$ whenever $n \equiv 2(\bmod 4)$. However, $\mu_{k}(n)$ is large enough on the average.

Theorem 6.5. For all $n, k$ with $2 \leq k \leq n$ there exists an $m$ such that $n-k+2 \leq m \leq n$ and $\left|\mu_{k}(m)\right|>(m-1) !(k-1)^{-m-1}$.

Proof. (The argument is a special case of Turán's principle [Tu].) Let $\alpha_{1}, \ldots, \alpha_{k-1}$ be the roots of $p_{k}(x)$. We have $\min _{i}\left|\alpha_{i}\right|<(k-1)$, since 
$\prod_{i} \alpha_{i}= \pm(k-1)$ !. Assume that $\left|\alpha_{1}\right|<k-1$. Write

$$
q(x)=\prod_{i=2}^{k-1}\left(x-\alpha_{i}\right)=\sum_{j=0}^{k-2} b_{j} x^{j} .
$$

Using (4.3) we get

$$
\begin{aligned}
\sum_{j=0}^{k-2} b_{j} \frac{\mu_{k}(n-j)}{(n-j-1) !} & =-\sum_{j=0}^{k-2} \sum_{i=1}^{k-1} b_{j} \alpha_{i}^{-n+j}=-\sum_{i=1}^{k-1} q\left(\alpha_{i}\right) \alpha_{i}^{-n} \\
& =-q\left(\alpha_{1}\right) \alpha_{1}^{-n}=-(k-1) ! p_{k}^{\prime}\left(\alpha_{1}\right) \alpha_{1}^{-n}=\alpha_{1}^{-n+k-1} .
\end{aligned}
$$

Hence there is a $j, 0 \leq j \leq k-2$, such that

$$
\left|b_{j} \frac{\mu_{k}(n-j)}{(n-j-1) !}\right|>\frac{1}{k-1}\left|\alpha_{1}\right|^{-n+k-1} .
$$

One can show by induction (using the Horner scheme) that

$$
b_{j}=-(k-1) ! \alpha_{1}^{-j-1} p_{j}\left(\alpha_{1}\right),
$$

and hence

$$
\begin{aligned}
\left|b_{j}\right| & =\left|\alpha_{1}\right|^{-j-1}(k-1) !\left|\left(p_{k}\left(\alpha_{1}\right)-p_{j}\left(\alpha_{1}\right)\right)\right| \\
& =\left|\alpha_{1}\right|^{-j-1}\left|\alpha_{1}^{k-1}+(k-1) \alpha_{1}^{k-2}+\cdots+(k-1)(k-2) \cdots(j+1) \alpha_{1}^{j}\right| \\
& \leq(k-1)^{k-j}\left|\alpha_{1}\right|^{-1} .
\end{aligned}
$$

Thus

$$
\begin{aligned}
\left|\mu_{k}(n-j)\right| & >\frac{1}{k-1}(n-j-1) !\left|\alpha_{1}\right|^{-n+k-1}\left|b_{j}\right|^{-1} \\
& \geq(n-j-1) !\left|\alpha_{1}\right|^{-n+k}(k-1)^{-k+j-1} \\
& >(n-j-1) !(k-1)^{-n+j-1} .
\end{aligned}
$$

Proof of Theorem 6.1. I. The first bounds for both the size and the depth follow by elementary considerations. Consider any linear decision tree $T$ for the $k$ equal problem; we may assume that it does not carry out unnecessary tests; i.e., there are both YES-leaves and NO-leaves below every internal node.

For every subset $1 \leq i_{1}<\cdots<i_{k} \leq n$ of indices, consider an input with $x_{i_{1}}=\cdots=x_{i_{k}}=0$ but having remaining entries all different from 0 and each other. Such inputs corresponding to different subsets of indices must go to different leaves, since the set of inputs going to any leaf is convex and the line segment connecting any two of these YES instances contains a NO instance. Hence the number of YES-leaves is at least $\left(\begin{array}{l}n \\ k\end{array}\right)$.

The cell corresponding to a YES-leaf is contained in $V_{\mathscr{S}_{n, k}}$, and so it has dimension at most $n-k+1$. Hence we must have used at least $k-1$ tests resulting in equality along the path to any YES-leaf.

It is also easy to see that $M_{\mathscr{A}_{n, k}}$ cannot contain any $k$-dimensional affine subspace: elements of such a subspace would have the form $b+\lambda_{1} a_{1}+\cdots+\lambda_{k} a_{k}$ 
for some fixed vectors $b, a_{1}, \ldots, a_{k}$ (with $a_{1}, \ldots, a_{k}$ linearly independent), and it is trivial to choose the $\lambda_{i}$ so that at least $k$ entries are 0 . Hence if we consider a NO-leaf of $T$, the corresponding cell cannot contain a $k$-dimensional affine subspace, and therefore we must have at least $n-k+1$ nodes on the path leading to it.

Consider any NO-leaf $w$ with an $n$-dimensional cell, and let $w^{\prime}$ be its father. As remarked, there must be a YES-leaf $w^{\prime \prime}$ somewhere below $w^{\prime}$, and so there must be at least $k-1$ tests resulting in equality along the path to $w^{\prime \prime}$. But none of the tests along the path leading to $w^{\prime}$ give equality (else, $w$ would not be $n$-dimensional), so we must have at least $(n-k)+(k-1)=n-1$ tests. Thus we have shown that $\operatorname{depth}(T) \geq n-1$.

II. It seems that the estimates obtained in part I are about as far as elementary arguments will take us. They do prove the theorem for $k \geq n / 3$. For the general case, however, we have to make use of the techniques developed in the earlier sections. It suffices to prove the lower bound on the size, which implies the lower bound on the depth. Assume that $k<n / 2$.

One possibility is to use Theorem 6.5. Choosing $n \geq m \geq n-k+2$ as in Theorem 6.5, we get

$$
\begin{aligned}
\ell_{k}^{-}(n) & \geq \ell_{k}^{-}(m) \geq\left|\mu_{k}(m)\right| \geq(m-1) !(k-1)^{-m-1} \\
& \geq(n-k+1) !(k-1)^{-n+k-3} .
\end{aligned}
$$

(The last inequality uses that $v !(k-1)^{-v}$ is increasing as a function of $v$ for $v \geq k-1$.) Hence

$$
\ell_{k}^{-}(n) \geq \frac{1}{(k-1)^{2}}\left(\frac{n-k+1}{3(k-1)}\right)^{n-k+1}>\left(\frac{n}{8 k}\right)^{n / 2} .
$$

This bound gives the right order of magnitude for the depth, but we can get a better bound by using the inequality $\ell_{k}^{-}(n) \geq \sum_{i} \tilde{\beta}_{i}\left(\Pi_{n, k}\right)$. An explicit formula for $\tilde{\beta}_{i}\left(\Pi_{n, k}\right)$ appears in Proposition 4.9. We will extract one term from the sum expressing an appropriate Betti number. More precisely, letting $t=\left\lfloor\frac{n}{2 k}\right\rfloor$ and $i_{0}=i_{1}=\cdots=i_{t-1}=0$, we get

$\ell_{k}^{-}(n) \geq(t-1) !\left(\begin{array}{l}n-1 \\ k-1\end{array}\right)\left(\begin{array}{c}n-k-1 \\ k-1\end{array}\right)\left(\begin{array}{c}n-2 k-1 \\ k-1\end{array}\right) \cdots\left(\begin{array}{c}n-(t-1) k-1 \\ k-1\end{array}\right) \cdot t^{n-t k}$.

Here each factor in the numerators of the binomial coefficients is at least $n / 2$, while the denominator can be estimated from above by $(k / 2)^{(k-1) t}$. Hence we get (assuming that $k \geq 3$ to exclude trivialities)

$$
\begin{aligned}
\ell_{k}^{-}(n) & \geq(t-1) !\left(\frac{n}{k}\right)^{(k-1) t} t^{n-t k}=t^{n}\left(\frac{n}{k t}\right)^{(k-1) t}(t-1) ! t^{-t} \\
& \geq\left(\frac{n}{3 k}\right)^{n} 2^{(k-1) t}(t-1) ! t^{-t}>\left(\frac{n}{3 k}\right)^{n} .
\end{aligned}
$$

$6 \mathrm{~b}$. The $k$-unequal problem. "Given real numbers $x_{1}, x_{2}, \ldots, x_{n}$, decide whether $k$ of them are pairwise different."

The case $k=2$ is fairly simple to settle by elementary considerations and is not discussed here. So we assume that $k \geq 3$. 
It is easy to solve this problem by a decision tree of depth $O(n \log k)$. The idea is to sort the numbers by the following insertion method. We process $x_{1}, x_{2}, \ldots$, one-by-one. We maintain an ordered list $\left(y_{1}, \ldots, y_{r}\right)$ of the distinct $x_{i}$ we have found after processing $x_{1}, \ldots, x_{m}$. If $r \geq k$, we stop; we know that the answer to the $k$-unequal-problem is YES. If $r<k$, then we process $x_{m+1}$ by inserting it into the list $\left(y_{1}, \ldots, y_{r}\right)$ by binary search. This takes $\lceil\log (r+1)\rceil<\log (2 k)$ comparisons. If we find that $x_{m+1}$ is already in the list, we throw it out; else, we insert it. Clearly this procedure requires at most $n \log (2 k)$ comparisons; i.e., it gives a decision tree of depth $n \log (2 k)$.

We prove that this tree is optimal up to a constant factor, even using the more powerful model of linear decision trees.

Theorem 6.6. Every linear decision tree for the $k$-unequal problem has size at least

$$
\max \left\{\left(\begin{array}{c}
n \\
k-2
\end{array}\right),(k-1)^{n} 3^{1-k}\right\}
$$

and depth at least

$$
\max \left\{n-1, n \log _{3}(k-1)-k+1\right\} .
$$

The proof is analogous to that of Theorem 6.1, but in many respects easier. The $k$-unequal problem is a special case of the problem of testing membership in the complement of a subspace arrangement. For each partition $\pi$ of $[n]$ into $k-1$ classes, let $B_{\pi}$ denote the linear subspace of $\mathbb{R}^{n}$ of dimension $k-1$ given by the equations $x_{i}=x_{j}$ whenever $i$ and $j$ belong to the same class of $\pi$. Let $\mathscr{B}_{n, k}$ denote the arrangement in $\mathbb{R}^{n}$ of the $S(n, k-1)$ subspaces $B_{\pi}$. It is clear that the $k$-unequal problem is to decide whether $x \in M_{\mathscr{B}_{n, k}}$ for points $x \in \mathbb{R}^{n}$. Therefore by Theorem 3.7, we can use the Möbius function of the corresponding intersection lattice to obtain a lower bound for the size of linear decision trees for this problem. Again, the intersection lattice has a nice combinatorial description, which is seen as in the proof of Proposition 6.2.

Proposition 6.7. The intersection lattice of $\mathscr{B}_{n, k}, 2 \leq k \leq n$, is isomorphic to the lattice $\Pi_{n}^{k-1}$. If under this isomorphism a subspace $x \in \mathscr{B}_{n, k}$ corresponds to a partition in $\Pi_{n}^{k-1}$ with $j$ blocks, then $\operatorname{dim}(x)=j$.

From this, Corollary 3.8 and Theorem 5.3 we get

Theorem 6.8.

$$
\chi\left(M_{\mathscr{B}_{n, k}}\right)=1+(-1)^{n-k} \sum_{r=1}^{k-1} r ! r S(n-k+r, r) .
$$

The lattice $\Pi_{n}^{k-1}$ is a lower truncation of the geometric lattice $\Pi_{n}$. Using that shellability of geometric lattices is preserved under such truncation (see $[B 1,(11.13)])$, and that shellability implies vanishing of Betti numbers below the top dimension in all intervals [B1, $\S 11]$, one can deduce from Proposition 3.4 that $M_{\mathscr{B}_{n, k}}$ has nonvanishing Betti numbers only in dimensions 0 and 
$n-k$. Hence, we have

$$
\sum_{i} \beta_{i}\left(M_{\mathscr{B}_{n, k}}\right)=1+\sum_{r=1}^{k-1} r ! r S(n-k+r, r),
$$

which shows that Euler characteristic and sum of Betti numbers give the same complexity lower bound in this case.

We obtain the following bounds from Theorems 2.1 and 6.8.

Corollary 6.9. Let $T$ be a linear decision tree for the $k$-unequal problem for $n$ numbers. Then both the number of YES-leaves and the number of NO-leaves of $T$ are at least

$$
\sum_{r=1}^{k-1} r ! r S(n-k+r, r)
$$

Proof of Theorem 6.6. Considering the last term in Corollary 6.9, and using the trivial inequality

$$
S(n, k)>k^{n-k},
$$

we get that the number of YES-leaves in any linear decision tree is at least

$$
(k-1) !(k-1)^{n-k+1}=(k-1)^{n} \frac{(k-1) !}{(k-1)^{k-1}}>(k-1)^{n} 3^{1-k} .
$$

A lower bound for depth follows by taking the logarithm.

For the remaining bounds we can argue as in part I of the proof of Theorem 6.1. Let $T$ be a linear tree testing for membership in $M_{\mathscr{B}_{n, k}}$. A cell corresponding to a NO-leaf is contained in $V_{\mathscr{B}_{n, k}}$, so it has dimension at most $k-1$. Hence along the path to any NO-leaf we must have used at least $n-k+1$ tests resulting in equality.

Also, $M_{\mathscr{B}_{n, k}}$ cannot contain any $(n-k+2)$-dimensional affine subspace: elements of such a subspace would have the form $b+\lambda_{1} a_{1}+\cdots+\lambda_{n-k+2} a_{n-k+2}$ for some fixed vectors $b, a_{1}, \ldots, a_{n-k+2}$ (with $a_{1}, \ldots, a_{n-k+2}$ linearly independent), and the $\lambda_{i}$ 's can clearly be chosen so that at least $n-k+2$ entries are equal, implying that the point lies in $V_{\mathscr{B}_{n, k}}$. Hence if we consider a YES-leaf of $T$, the corresponding cell cannot contain a $(n-k+2)$-dimensional affine subspace, and therefore we must have at least $k-1$ nodes on the path leading to it.

Now consider any YES-leaf $w$ with an $n$-dimensional cell, and let $w^{\prime}$ be its father. There must be a NO-leaf $w^{\prime \prime}$ somewhere below $w^{\prime}$ (unless the tree $T$ is redundant), and so there must be at least $n-k+1$ tests resulting in equality along the path to $w^{\prime \prime}$. But none of the tests along the path leading to $w^{\prime}$ give equality (otherwise $w$ would not be $n$-dimensional), so we must have at least $(n-k+1)+(k-1)-1=n-1$ tests. Thus we have shown that $\operatorname{depth}(T) \geq n-1$.

For every subset $1 \leq i_{1}<\cdots<i_{n-k+2} \leq n$ of indices, consider an input with $x_{i_{1}}=\cdots=x_{i_{n-k+2}}=0$ but having remaining entries all different from 0 and from each other. Such inputs corresponding to different subsets of indices must go to different NO-leaves, since the set of inputs going to any leaf is convex 
and the line segment connecting any two of these NO instances contains a YES instance. Hence the number of NO-leaves is at least $\left(\begin{array}{c}n \\ n-k+2\end{array}\right)$.

6c. The $k$-divisibility problem. "Given real numbers $x_{1}, x_{2}, \ldots, x_{n}$, decide whether the number of $x_{i}$ 's equal to any given real number is divisible by $k$." $n / 2$.

We suppose that $k$ divides $n$, and set $t=n / k$. Assume also that $2 \leq k \leq$

It is again easy to solve this problem by a decision tree of depth $O(n \log (n / k))$. Just as for the $k$-equal problem, we find the $k$ th, $(2 k)$ th, $\ldots,(t k)$ th largest elements, and the blocks between them, in $O(n \log (n / k))$ comparisons. Then we compare each of these elements with the elements in the block preceding it. If we find equality in all these comparisons, we conclude that the multiplicity of each element is divisible by $k$; else, we conclude that it is not.

The following result shows that this tree is essentially best possible.

Theorem 6.10. Every linear decision tree for the $k$-divisibility problem has size at least

and depth at least

$$
\left(\frac{n}{k+4 \ln k}\right)^{n}
$$

$$
n \log _{3} \frac{n}{k+4 \ln k} \text {. }
$$

Similarly as before, we use our general topological bound. Let $\mathscr{D}_{n, k}$ denote the set of all linear subspaces $B_{\pi}$, where $\pi$ is a partition of $[n]$ into classes with cardinality divisible by $k$; then the $k$-divisibility problem is just testing membership in $\mathscr{D}_{n, k}$. The intersection lattice corresponding to this arrangement has the following combinatorial description:

$$
\Pi_{n}^{(k)}=\left\{\pi \in \Pi_{n} \text { : all block sizes in } \pi \text { are divisible by } k\right\} \cup\{\hat{0}\} .
$$

To apply our topological methods, we have to compute the Möbius function $\mu_{n}^{(k)}$ of this lattice. Fortunately, we can just quote the following result of Stanley [S1, S2]. Define the descent set of a permutation $\sigma \in S_{n}$ to be

$$
\{i \in[n-1]: \sigma(i)>\sigma(i+1)\} \text {. }
$$

Proposition 6.11. $\left|\mu_{n}^{(k)}(\hat{0}, \hat{1})\right|$ is the number of permutations of $n-1$ elements with descent set $\{k, 2 k, \ldots, n-k\}$.

From this combinatorial interpretation it is easy to derive the following inequality.

Proposition 6.12.

$$
\left|\mu_{n}^{(k)}(\hat{0}, \hat{1})\right|>\frac{(n-t) !(t-1) !}{((k-1) !)^{t}} .
$$

Proof. Partition the elements $\{1, \ldots, n-t\}$ into $t$ classes $A_{1}, \ldots, A_{t}$ of size $k-1$. Consider the permutation that starts with the elements of $A_{1}$ in increasing order, followed by any element of $\{n-t+1, \ldots, n-1\}$; then come the elements of $A_{2}$ in increasing order, followed by any other element of $\{n-t+1, \ldots, n-1\}$, etc. (We end with the elements of $A_{t}$ in increasing 
order). It is clear that this permutation has the right descent set. The number of permutations arising this way is the lower bound on the right-hand side.

Using Theorem 3.7 and Proposition 6.12, we obtain the following bound.

Corollary 6.13. Let $T$ be a linear decision tree for the $k$-divisibility problem for $n$ numbers. Then both the number of YES-leaves and the number of NO-leaves of $T$ are at least

$$
\frac{(n-t) !(t-1) !}{(k-1) !^{t}}
$$

Hence the proof of Theorem 6.10 consists of estimating these factorials as before, and is omitted.

\section{NOTE ADDED IN PROOF (FEBRUARY 1994)}

The Betti number lower bounds have been extended to algebraic decision trees and algebraic computation trees in recent work by A. C.-C. Yao.

\section{REFERENCES}

[BO] M. Ben-Or, Lower bounds for algebraic computation trees, Proc. 15th Annual ACM Sympos. on Theory of Computing, ACM Press, New York, 1983, pp. 80-86.

[B1] A. Björner, Topological methods, Handbook of Combinatorics (R. Graham, M. Grötschel, and L. Lovász, eds.), North-Holland, Amsterdam, 1994.

[B2] - Subspace arrangements, Proc. 1st European Congress Math. (Paris, 1992) (A. Joseph and R. Rentschler, eds.), Birkhäuser, Basel-Boston, 1994.

[BK] A. Björner and G. Kalai, An extended Euler-Poincaré theorem, Acta Math. 161 (1988), 279-303.

[BLVŽ] A. Björner, L. Lovász, S. Vrećica, and R. Živaljević, Chessboard complexes and matching complexes, J. London Math. Soc. (to appear).

[BLY] A. Björner, L. Lovász, and A. C.-C. Yao, Linear decision trees: volume estimates and topological bounds, Proc. 24th Annual ACM Sympos. on Theory of Computing, ACM Press, New York, 1992, pp. 170-177.

[BW] A. Björner and V. Welker, The homology of " $k$-equal" manifolds and related partition lattices, Adv. in Math. (to appear).

[CHR] A. R. Calderbank, P. Hanlon, and R. W. Robinson, Partitions into even and odd block size and some unusual characters of the symmetric groups, Proc. London Math. Soc. (3) 53 (1986), 288-320.

[DL] D. Dobkin and R. Lipton, On the complexity of computations under varying sets of primitives, Automata Theory and Formal Languages (H. Bradhage, ed.), Lecture Notes in Comput. Sci., vol. 33, Springer-Verlag, New York and Berlin, 1975, pp. 110-117.

[GM] M. Goresky and R. MacPherson, Stratified Morse theory, Ergeb. Math. Grenzgeb. (3), Band 14, Springer-Verlag, Berlin, 1988.

[MH] F. Meyer auf der Heide, A polynomial linear search algorithm for the n-dimensional knapsack problem, J. Assoc. Comput. Mach. 31 (1984), 668-676.

[Mi] J. Milnor, On the Betti numbers of real algebraic varieties, Proc. Amer. Math. Soc. 15 (1964), 275-280.

[Mu] J. R. Munkres, Elements of algebraic topology, Addison-Wesley, Menlo Park, 1984.

[O] O. A. Oleinik, Estimates of the Betti numbers of real algebraic hypersurfaces, Math. Sb. 28 (1951), 635-640. 
[OP] O. A. Oleinik and I. B. Petrovsky, On the topology of real algebraic surfaces, Izv. Akad. Nauk SSSR 13 (1949), 389-402; English transl., Amer. Math. Soc. Transl. Ser. 1, vol. 7, Amer. Math. Soc., Providence, RI, 1962, pp. 399-417.

[R] E. M. Reingold, Computing the maxima and the median, Proc. 12th IEEE Annual Sympos. on Switching and Automata Theory, IEEE, Piscataway, NJ, 1971, pp. 216-218.

[S1] R. P. Stanley, Binomial posets, Möbius inversion and permutation enumeration, J. Combin. Theory Ser. A 20 (1976), 336-356.

[S2] — Exponential structures, Stud. Appl. Math. 59 (1978), 73-82.

[S3] _ Enumerative combinatorics, Vol. 1, Wadsworth \& Brooks/ Cole, Monterey, CA, 1986.

[SY] M. Steele and A. Yao, Lower bounds for algebraic decision trees, J. Algorithms 3 (1982), 1-8.

[Sz] G. Szegö, Über eine Eigenschaft der Exponentialreihe, Sitzungsber. Berlin Math. Gesellschaft 23 (1924), 50-64.

[Th] R. Thom, Sur l'homologie des variétés algébriques réelles, Differential and Algebraic Topology (S. S. Cairns, ed.), Princeton Univ. Press, Princeton, NJ, 1965.

[Tu] P. Turán, Eine neue Methode in der Analysis und deren Anwendungen, Akad. Kiadó, Budapest, 1953.

[V] R. S. Varga, Scientific computation on mathematical problems and conjectures, CBMS-NSF Regional Conf. Ser. in Appl. Math., vol. 60, SIAM, Philadelphia, PA, 1990.

[Y] A. C.-C. Yao, Algebraic decision trees and Euler characteristics, Proc. 33rd Annual IEEE Sympos on Foundations of Computer Science, October 1992, pp. 268-277.

[ZŽ] G. M. Ziegler and R. T. Živaljević, Homotopy types of arrangements via diagrams of spaces, Math. Ann. 295 (1993), 527-548.

ABSTRACT. Topological methods are described for estimating the size and depth of decision trees where a linear test is performed at each node. The methods are applied, among others, to the questions of deciding by a linear decision tree whether given $n$ real numbers (1) some $k$ of them are equal, or (2) some $k$ of them are unequal. We show that the minimum depth of a linear decision tree for these problems is at least (1) $\max \left\{n-1, n \log _{3}(n / 3 k)\right\}$, and (2) $\max \left\{n-1, n \log _{3}(k-1)-k+1\right\}$.

Our main lower bound for the size of linear decision trees for polyhedra $P$ in $\mathbb{R}^{n}$ is given by the sum of Betti numbers for the complement $\mathbb{R}^{n} \backslash P$. The applications of this general topological bound involve the computation of the Möbius function of intersection lattices of certain subspace arrangements. In particular, this leads to computing various expressions for the Möbius function of posets of partitions with restricted block sizes. Some of these formulas have topological meaning. For instance, we derive a formula for the Euler characteristic of the subset of $\mathbf{R}^{n}$ of points with no $k$ coordinates equal in terms of the roots of the truncated exponential $\sum_{i<k} x^{i} / i$ !

Department of Mathematics, Royal Institute of Technology, Stockholm, Sweden S-100 44

Department of Computer Science, Eötvös LoRÁnd University, BudAPEST, Hungary H1088

Current address: Department of Mathematics, Yale University, New Haven, Connecticut 06520 